\title{
Article
}

\section{Isolation of Small Extracellular Vesicles from Human Sera}

\author{
Małgorzata S. Małys ${ }^{1} \mathbb{D}^{\mathrm{D}}$, Christof Aigner ${ }^{1,2}{ }^{\mathbb{D}}$, Stefan M. Schulz ${ }^{1}$, Helga Schachner ${ }^{1}$, Andrew J. Rees ${ }^{1}$ and \\ Renate Kain 1,*(D)
}

1 Department of Pathology, Medical University Vienna, 1090 Vienna, Austria; malgorzata.malys@meduniwien.ac.at (M.S.M.); christof.aigner@meduniwien.ac.at (C.A.); stefan.schulz@meduniwien.ac.at (S.M.S.); helga.schachner@meduniwien.ac.at (H.S.); andrew.rees@meduniwien.ac.at (A.J.R.)

2 Division of Nephrology and Dialysis, Department of Medicine III, Medical University Vienna, 1090 Vienna, Austria

* Correspondence: renate.kain@meduniwien.ac.at

check for updates

Citation: Małys, M.S.; Aigner, C.; Schulz, S.M.; Schachner, H.; Rees, A.J.; Kain, R. Isolation of Small Extracellular Vesicles from Human Sera. Int. J. Mol. Sci. 2021, 22, 4653. https://doi.org/10.3390/ijms 22094653

Academic Editor: Maria Pascual

Received: 1 April 2021

Accepted: 26 April 2021

Published: 28 April 2021

Publisher's Note: MDPI stays neutral with regard to jurisdictional claims in published maps and institutional affiliations.

Copyright: (c) 2021 by the authors. Licensee MDPI, Basel, Switzerland. This article is an open access article distributed under the terms and conditions of the Creative Commons Attribution (CC BY) license (https:// creativecommons.org/licenses/by/ $4.0 /)$.

\begin{abstract}
Robust, well-characterized methods for purifying small extracellular vesicles (sEV) from blood are needed before their potential as disease biomarkers can be realized. Here, we compared isolation of sEV from serum by differential ultracentrifugation (DUC) and by exclusion chromatography using commercially available Exo-spin ${ }^{\mathrm{TM}}$ columns. We show that sEV can be purified by both methods but Exo-spin ${ }^{\mathrm{TM}}$ columns contain copious additional particles recorded by nanoparticle tracking analysis, invalidating its use for quantifying yields. DUC samples contained higher concentrations of exosome specific proteins CD9, CD63 and CD81 and electron microscopy confirmed that most particles in DUC preparations were sEV, whereas Exo-spin ${ }^{\mathrm{TM}}$ samples also contained copious co-purified plasma lipids. MACSPlex bead analysis identified multiple exosome surface proteins, with stronger signals in DUC samples, enabling detection of 21 of 37, compared to only 10 in Exo-spin ${ }^{\mathrm{TM}}$ samples. Nevertheless, the pattern of expression was consistent in both preparations, indicating that lipids do not interfere with bead-based technologies. Thus, both DUC and Exo-spin ${ }^{\mathrm{TM}}$ can be used to isolate sEV from human serum and what is most appropriate depends on the subsequent use of sEV. In summary, Exo-spin ${ }^{\mathrm{TM}}$ enables isolation of sEV from blood with vesicle populations similar to the ones recovered by DUC, but with lower concentrations.
\end{abstract}

Keywords: extracellular vesicles; exosomes; purification; isolation

\section{Introduction}

Extracellular vesicles (EV) are a heterogeneous group of a lipid bound structures released from cells into the surrounding medium [1]. The number and composition of EV depends on the stress and activation status of the originating cell, and this provides the basis for their critical role in cell-cell communication during homeostasis and after injury. They have often been isolated from tissue culture supernatants and less commonly from the blood of donors, including those with systemic disease, in whom they are especially abundant. This raises the possibility of using blood derived EV to probe the pathogenesis of disease, as biomarkers of its activity, and even as novel vehicles to deliver therapy.

EV can be classified into three distinct types, defined by their biogenesis [1]: apoptopic bodies whose diameters range between 100-5000 nm, and which originate as blebs that detach from the surface of apoptotic cells; microvesicles, with diameters of 100-1000 nm, which are formed by budding out from the plasma membrane; and exosomes, of 30-150 nm, which are generated as intraluminal vesicles in late endosomes that fuse with the plasma membrane to release the ILV into the extracellular fluid as exosomes [2]. Despite their differing origins, the three species of EV have overlapping physical properties and share some of the molecules expressed on their limiting membranes, and also components of the cargo they carry. These overlapping properties have hampered the development of strategies to obtain pure preparations of the individual types of $\mathrm{EV}$, and for this reason 
the International Society for Extracellular Vesicles, recommends that purified exosome fractions be simply designated as small EVs (sEV).

Exosomes have been subjected to intense research because their cargo is selectively internalized, and includes specific proteins and miRNA, which they deliver to adjacent and more distant cells $[3,4]$. The exosome cargo reflects the type and state of the cells, from which they originate, as do the molecules expressed in its limiting membrane. Some of the surface molecules (such as CD9, CD81 and CD63) are accepted as characteristic, if not completely exclusive, exosome markers that are expressed on most exosomes, regardless of origin. Other proteins, not specific for exosomes, are restricted to sEV derived from particular cell lineages, such as the asialoglycoprotein receptor on hepatocyte-derived exosomes [5], and CD31 and CD146 on those released by endothelial cells [6]. Finally, some molecules, such as Major Histocompatibility Complex Class I and Class II molecules, are expressed on EV membranes more generally $[4,7,8]$. It follows that characterizing exosomes in human blood, and other biological fluids, could provide insights into the nature and activity of systemic disease. However clinical application of this approach requires simple and robust methods for isolating exosomes from human blood, which is challenging $[1,8,9]$.

Numerous techniques have been used to isolate exosomes from tissue culture supernatants [10-20], but purifying them from human blood is more difficult for at least two reasons: the EV in blood are far more heterogeneous than those generated in vitro, and second, they are suspended in plasma, which is more complex than tissue culture medium. Differential ultracentrifugation (DUC) is currently the standard method for isolating exosomes relatively free from contamination from other EV [20-23], but is cumbersome and requires relatively large sample volumes. The search for simpler and quicker methods led to strategies using size exclusion chromatography, such as commercially available Exo-spin ${ }^{\mathrm{TM}}$ columns (Cell Guidance Systems Ltd., Cambridge, UK) $[17,24]$. These isolate exosomes from culture supernatants are efficient and are simple enough for use in the clinic. It is now important to determine how efficiently and with what purity Exo-spin ${ }^{\mathrm{TM}}$ (EX05) columns purify exosome-containing sEV from human blood, and to compare results to isolation by DUC, which is what we have done.

We purified serum sEV from normal human donors by DUC and Exo-spin ${ }^{\mathrm{TM}}$ columns and compared the resulting exosome-rich fractions. Immunoblotting, ELISA and MACSPlex bead assays for CD9, CD63 and CD81 showed that both methods efficiently isolate $\mathrm{sEV}$, and this was corroborated by particle counting by nanoparticle tracking analysis (NTA), which was 500-fold greater in the Exo-spin ${ }^{\mathrm{TM}}$ samples. Immuno-electron microscopy (EM) confirmed that the majority of sEV in the DUC were exosomes; by contrast, most particles in the Exo-spin ${ }^{\mathrm{TM}}$ samples co-purified lipoproteins and lipid droplets. Thus, Exospin ${ }^{\mathrm{TM}}$ columns effectively purify sEV from human serum, provided the influence of the attendant lipid contamination on the analytical strategies to be applied to the purified sEV is carefully considered.

\section{Results}

\subsection{Nanoparticle Tracking Analysis of Serum-Derived sEV Purified by DUC or Exo-spin ${ }^{\mathrm{TM}}$}

We quantified particles in the sEV preparations obtained by DUC or Exo-spin ${ }^{\mathrm{TM}}$ columns from nine fresh serum samples taken from six healthy donors. There were numerous particles in samples purified by both methods from all the sera (Supplementary Table S1). However, despite extraction from a 20 -fold larger serum volume ( $2 \mathrm{~mL}$ versus $0.1 \mathrm{~mL}$ ), there were far fewer particles in DUC preparations than in the Exo-spin ${ }^{\mathrm{TM}}$ ones: DUC-2.72 $\pm 2.22 \times 10^{8}$ (Range: $0.51-8.07 \times 10^{8}$ ) vs Exo-spin ${ }^{\mathrm{TM}}-49.76 \times 10^{8} \pm 76.95$ (Range: $\left.0.51-254.67 \times 10^{8}\right) ; p=0.03147$, Wilcoxon rank sum test (Figure 1a). After correcting for the different serum volumes used, there was a mean of 500-fold more particles isolated per $\mathrm{mL}$ of serum by Exo-spin ${ }^{\mathrm{TM}}$ than by DUC: $497.56 \pm 769.5 \times 10^{8} / \mathrm{mL}$ of serum and $0.99 \pm 1.11 \times 10^{8} / \mathrm{mL}$ of serum, respectively $\left(p=4.114 \times 10^{-5}\right.$, Wilcoxon rank sum test) (Figure 1b). Similarly, the size distribution was narrower (Figure 1e-g), and the particle mean diameter smaller (Figure 1c) in the Exo-spin ${ }^{\mathrm{TM}}$ preparations: Exo-spin ${ }^{\mathrm{TM}}$ 
$132.52 \pm 12.05 \mathrm{~nm}$; and DUC $-148.11 \pm 14.64 \mathrm{~nm}(p=0.0215$, Wilcoxon rank sum test). Typically, the diameter of exosomes is less than $150 \mathrm{~nm}$ and the proportion of particles of this size was significantly lower in the DUC preparations (Figure 1d): DUC $-57.66 \pm 8.8 \%$; Exo-spin ${ }^{\mathrm{TM}}-69.61 \pm 8.43 \%(p=0.03147$, Wilcoxon rank sum test). Consequently, the yield of small particles per ml of serum was significantly greater in Exo-spin ${ }^{\mathrm{TM}}$ purified samples than in DUC: Exo-spin ${ }^{\mathrm{TM}}-404.41 \pm 690.94 \times 10^{8}$; DUC—0.57 $\pm 0.64 \times 10^{8}$ $\left(p=4.114 \times 10^{-5}\right.$, Wilcoxon rank sum test). This suggests that Exo-spin ${ }^{\mathrm{TM}}$ purification is much more efficient than serum, but this needed to be verified by comparing the abundance of the exosome-specific proteins in the preparations, such as CD9, CD63 and CD81.

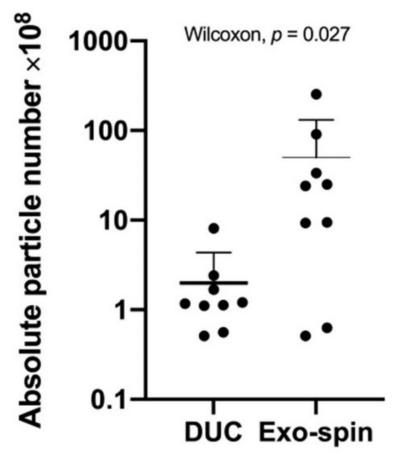

(a)

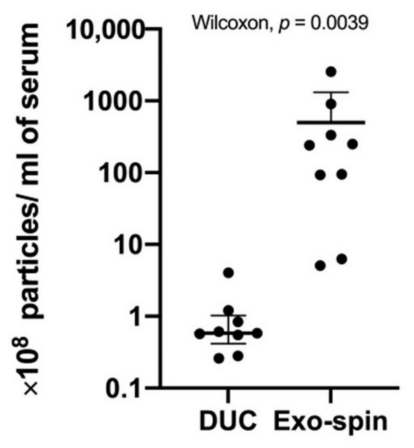

(b)

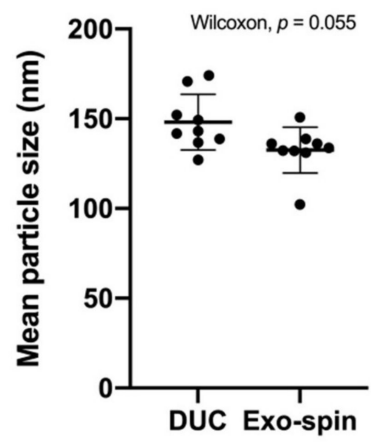

(c)

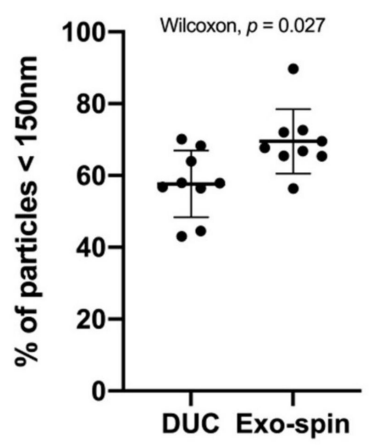

(d)

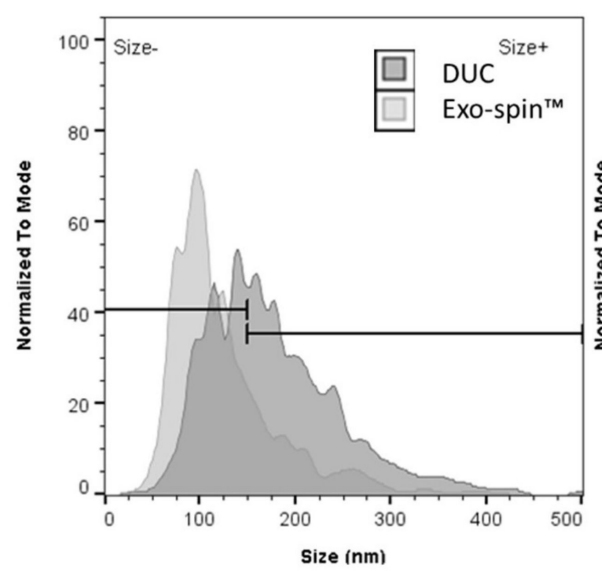

(e)

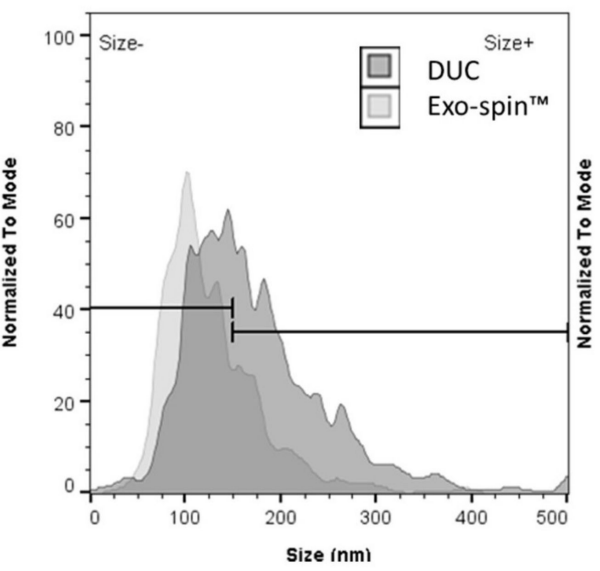

(f)

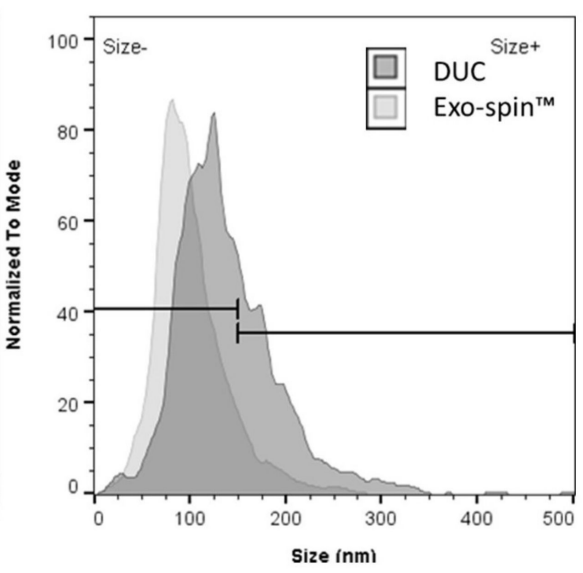

(g)

Figure 1. The mean particle number in SEV preparations isolated from nine serum samples withdrawn from 6 individuals. The graph (a) presents the total particle number in sEV suspensions isolated from different volumes of serum, respectively: Exo-spin ${ }^{\mathrm{TM}} 100 \mu \mathrm{l}$ and DUC $2000 \mu \mathrm{l}$; the mean particle number in sEV samples normalized to the volume of serum used for isolation is shown on graph (b); image (c) shows the mean size of vesicles in nanometers for DUC and Exo-spin ${ }^{\mathrm{TM}}$ preparations. Percentage of vesicles smaller than $150 \mathrm{~nm}$ is displayed on graph (d); representative size distribution of analyzed sEV preparations isolated by DUC and Exo-spin ${ }^{\mathrm{TM}}(\mathbf{e}-\mathbf{g})$. Statistical analysis was performed in R with Wilcoxon signed rank test for paired samples. The middle bar of each group shows the median with the error bars corresponding to the interquartile range. Abbreviations: DUC: differential ultracentrifugation.

\subsection{Analysis of the Abundance of Exosomal Markers by Western Blotting and ELISA}

Nanoparticle tracking analysis counts all the particles in a sample and not just EV, and we used the abundance of three characteristic exosome proteins, CD9, CD63 and CD81 as surrogates to estimate yields on sEV isolated from serum. In striking contrast to the NTA, immunoblotting showed that concentrations of CD9 and CD63 were appreciable higher in the DUC-purified sEV samples (Figure 2a,b and Supplementary Figure S1), suggesting that they contained more exosomes. Re-analysis of the samples by ELISA specific for 
CD9, CD63 and CD81 confirmed the result and showed the DUC preparations contained significantly higher mean concentrations of all three proteins (expressed in ELISA intensity units): CD9-0.96 \pm 0.48 and $0.18 \pm 0.07$ for DUC and Exo-spin ${ }^{\mathrm{TM}}$ respectively $(p=0.031$, Wilcoxon signed rank test); CD63 $-0.52 \pm 0.25$ and $0.16 \pm 0.07$ ( $p=0.031$, Wilcoxon signed rank test); and CD81-0.36 \pm 0.18 and $0.12 \pm 0.08$ ( $p=0.031$, Wilcoxon signed rank test). Results from an ELISA for calnexin excluded significant contamination of the samples with intracellular contents (data not shown). Concentrations of CD9, CD63 and CD81 varied from donor-to-donor, but were invariably higher in the DUC and correlated closely with each other, regardless of the purification method (Figure 2b-d). Consequently, ranking the donors based on the concentrations of CD9 gave near identical results to ranking them according to CD63 or CD81 (Figure 2b-d). The greater abundance of exosome specific proteins in DUC samples, together with the results from individual donors provide strong evidence that CD9, CD63 and CD81 concentrations in a given sample all reflect the number of exosomes that it contains.

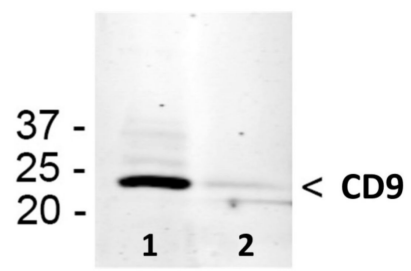

(a)

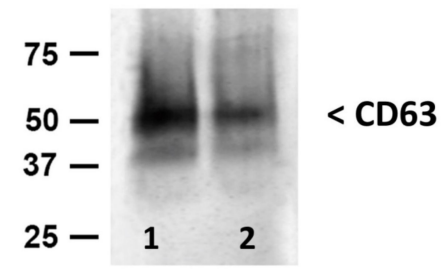

(b)
CD9

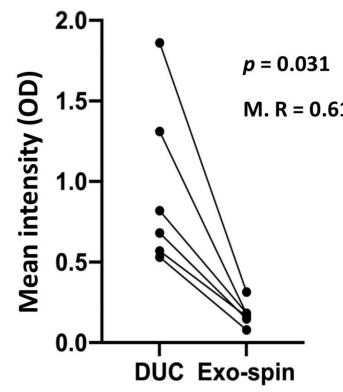

(c)
CD63

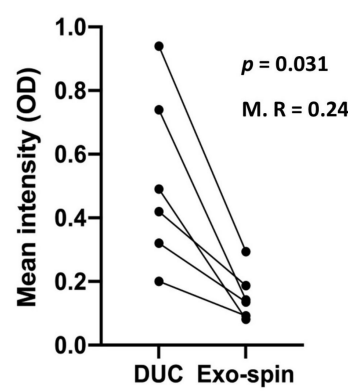

(d)
CD81

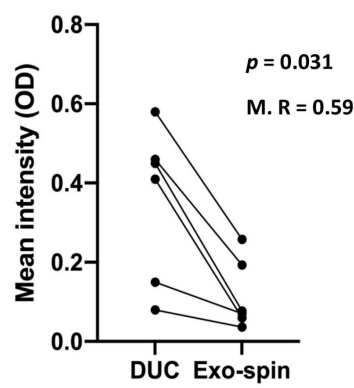

(e)

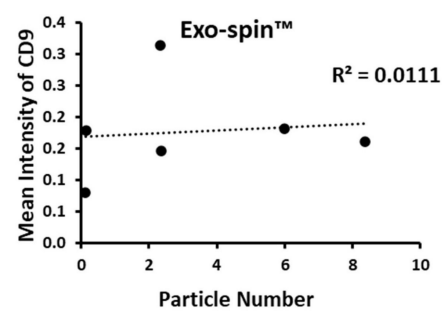

(g)

Figure 2. Image (a) shows a representative Western blot with antibody to CD9, and on a duplicate sample (image (b), to CD63 on small extracellular vesicles purified from one donor by either differential ultracentrifugation (lane 1) or Exo-spin ${ }^{\mathrm{TM}}$ (lane 2). ELISA results given in relative mean intensity (OD of measured absorbance $(490 \mathrm{~nm}-630 \mathrm{~nm})$ ) are shown on the axis y of graphs $(\mathbf{c}-\mathbf{e})$ for exosome specific markers (CD9, CD63, and CD81). Graphs present the mean intensity from 3 replicates of the small extracellular vesicle (sEV) preparations purified from six individuals by both methods-individual results of paired samples are connected by a line. Graphs $(\mathbf{f}, \mathbf{g})$ show the correlation between the $\mathrm{CD} 9$ mean intensity and particle numbers calculated by nanoparticle tracking analysis for DUC and Exo-spin ${ }^{\mathrm{TM}}$ samples, respectively. Statistical analysis was performed in $\mathrm{R}$ with a Wilcoxon signed rank test. Abbreviations: M. R = Multiply R. 
Next, we explored the obvious contradictions between NTA and protein abundance data by correlating the particle counts for individual donors with the concentration of CD9, CD63 and CD81 in the same sample. In the DUC preparations, the particle number correlated closely with the concentrations of all three exosome proteins: CD9- $\mathrm{R}^{2}=0.770$ $(p=0.021) ; C D 63-R^{2}=0.548(p=0.092) ; C D 81-R^{2}=0.256(p=0.305)$; whereas there was no correlation in the Exo-spin ${ }^{\mathrm{TM}}$ samples: CD9- $\mathrm{R}^{2}=0.011(p=0.842) ; \mathrm{CD} 63-\mathrm{R}^{2}=0.058$ $(p=0.644) ; C D 81-R^{2}=0.012(p=0.5)$ (Figure 2e-g and Supplementary Figure S2). These data strongly suggest that the Exo-spin ${ }^{\mathrm{TM}}$ purified samples contain large numbers of small particles that are not sEV and so we used electron microscopy to investigate their nature.

\subsection{Ultrastructure of sEV Preparations Isolated by Exo-spin ${ }^{\mathrm{TM}}$ and DUC and Analysed by Transmission Electron Microscopy (TEM)}

Samples from three donors were used to examine the ultrastructural appearances of sEV preparations purified by DUC and Exo-spin ${ }^{\mathrm{TM}}$. Many sEVs in the DUC samples had the distinctive cup shape previously reported to be characteristic of exosomes, but they were heterogenous in size and were often found in small groups with visible aggregates (Figure 3a,b). Immunostaining with gold-labelled specific antibodies showed that around $30 \%$ and $70 \%$ of the sEV expressed CD9 and CD63, respectively, confirming that they were exosomes (Figure 3c,e). The Exo-spin ${ }^{\mathrm{TM}}$ purified samples contained many more vesicles, which occurred in densely layered sheets and had a very different morphology to the DUC preparations. There were at least three distinct populations of particles (Figure 3d,f): dark vesicles with a high contrast membrane; smaller and brighter shade-like vesicles; and large vesicles with a single dark spot at one of the poles. Only rare vesicles expressed CD9 or CD63 in the immunogold stained preparations. These data confirm the inferences drawn from the protein expression data: in the DUC preparations, most of the particles identified by NTA are sEV. By contrast, the vast majority of vesicle-like particles purified by Exo-spin ${ }^{\mathrm{TM}}$ are not $\mathrm{sEV}$, and so we determined whether they could result from co-purified serum proteins or lipids.

\subsection{Co-Purified Lipoproteins Account for the Excess Particles in Exo-spin ${ }^{\mathrm{TM}}$ Preparations}

The mean total protein concentrations in DUC and Exo-spin ${ }^{\mathrm{TM}}$ preparations from five donors were $32.4 \mu \mathrm{g} / \mathrm{mL} \pm 7.79$ and $40.6 \mu \mathrm{g} / \mathrm{mL} \pm 16.81$, respectively. Silver stained SDS-PAGE of DUC samples showed a dominant band with the molecular mass, indicative of albumin, which equates to a concentration of less than $4.16 \mu \mathrm{g} / \mathrm{mL}$ if the designation is correct (Supplementary Figure S3). We did not detect albumin in the Exo-spin ${ }^{\mathrm{TM}}$ samples, but instead, the silver stained SDS-PAGE showed intense bands with masses $>250 \mathrm{kDa}$, which is suggestive of lipoproteins; there were no corresponding proteins identified in the DUC preparations (Supplementary Figure S3). Next, we measured concentrations of Apolipoprotein B (ApoB) by specific ELISA as a surrogate for lipoproteins more generally. The concentration of ApoB was similar in DUC and Exo-spin ${ }^{\mathrm{TM}}$ purified sEV samples, with $0.39 \pm 0.17$ and $0.4 \pm 0.23$ ELISA units, respectively. However, the ApoB concentration correlated closely with the NTA particle count in the Exo-spin ${ }^{\mathrm{TM}}$ samples $\left(\mathrm{R}^{2}=0.8538\right.$; $p=0.008)$, but not in those purified by DUC $\left(R^{2}=0.3561 ; p=0.211\right)$ (Figure $\left.4 a, b\right)$. Importantly, the serum triglyceride concentration correlated with the ApoB concentrations in the Exo-spin ${ }^{\mathrm{TM}} \mathrm{sEV}$ preparations $\left(\mathrm{R}^{2}=0.71 ; p=0.035\right)$, and with the NTA particle count $\left(R^{2}=0.945 ; p=0.001\right)$; there were similar, but less strong correlations between particle count and total serum cholesterol, HDL, LDL (Supplementary Figures S4-S7). None of these correlated in the DUC preparations $\left(R^{2}=0.003 ; p=0.91\right.$ and $R^{2}=0.135 ; p=0.473$, respectively; Figure 4c-f). 


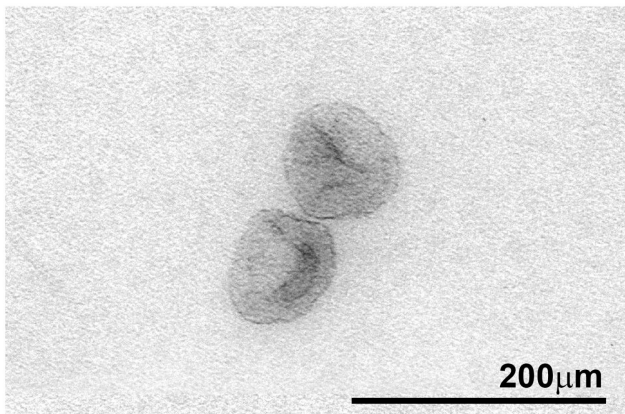

(a)

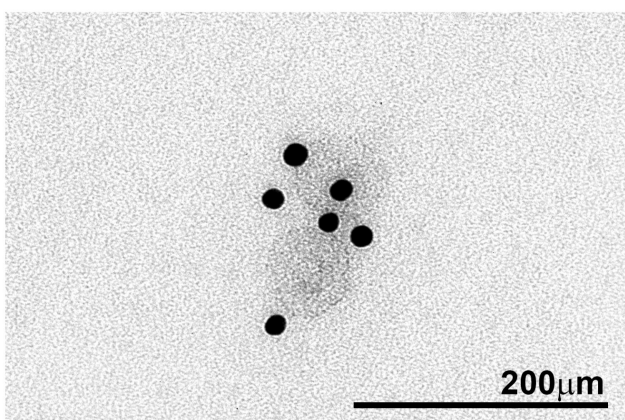

(c)

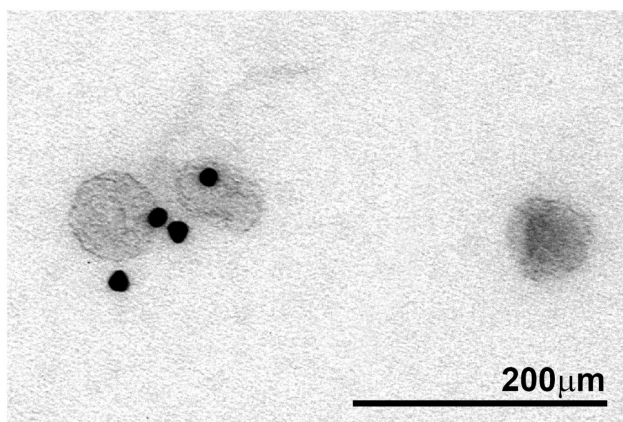

(e)

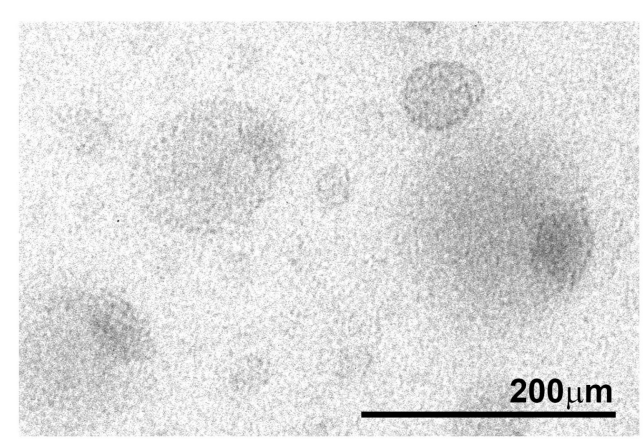

(b)

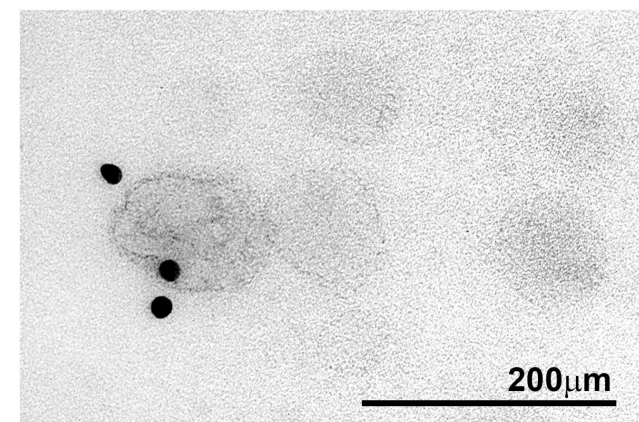

(d)

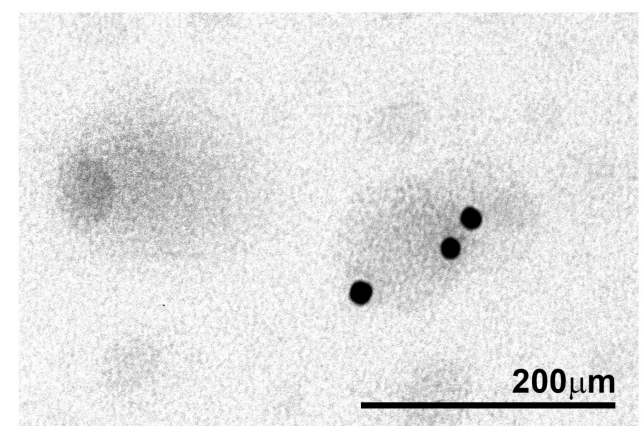

(f)

Figure 3. Transmission electron microscopy images of small extracellular vesicle (sEV) preparations obtained by two methods: differential ultracentrifugation $(\mathbf{a}, \mathbf{c}, \mathbf{e})$ and Exo-spin ${ }^{\mathrm{TM}}(\mathbf{b}, \mathbf{d}, \mathbf{f})$. sEV preparations were identified using antibodies specific to either CD9 $(\mathbf{c}, \mathbf{d})$ or CD63 (e,f) and antibody binding was confirmed by secondary antibodies conjugated to $15 \mathrm{~nm}$ gold particles.

These data strongly suggest that the excess particles in the Exo-spin ${ }^{\mathrm{TM}}$ samples result from co-purified triglyceride containing serum lipids, and this is supported by the EM images, since many of the particles are reminiscent of those reported for lipoproteins. In particular, those with the dark spot at one pole (Figure $5 b, d$ ) were strikingly similar to lipid droplets, in which triglycerides accumulate in a characteristic pocket-like structure, which has been described previously [25]. Immuno-EM using antibodies to ApoB revealed abundant gold particles in the Exo-spin ${ }^{\mathrm{TM}}$ preparations, whereas none of the vesicles in DUC preparations were ApoB positive. Unfortunately, the vesicles were too small and too abundant in the Exo-spin ${ }^{\mathrm{TM}}$ preparations to be certain, whether they were directly linked to the densely distributed gold particles (Figure 5). Nevertheless, we conclude that the excess particles in Exo-spin ${ }^{\mathrm{TM}}$ samples are serum lipoprotein complexes co-purified with the sEV, and it was important to know whether they influenced characterization of proteins expressed in the sEV limiting membrane. 


\section{DUC}

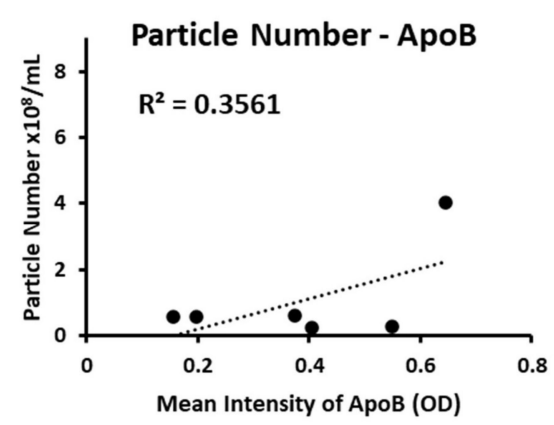

(a)

Trig - ApoB

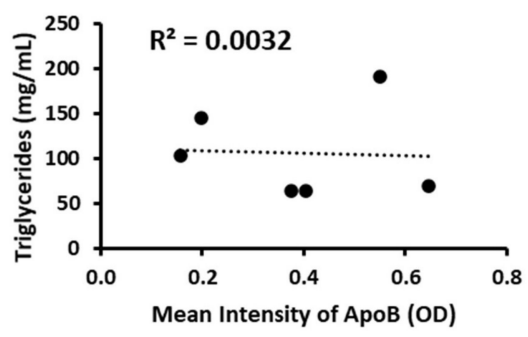

(c)

\section{Trig - Particle Number}

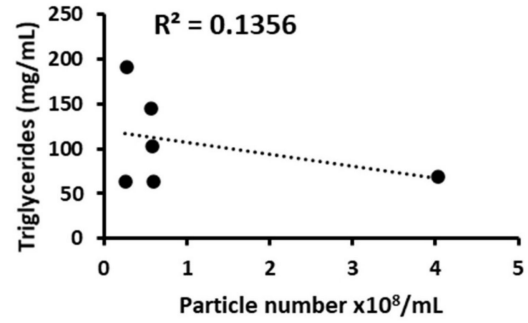

(e)

\section{Exo-spin $^{\mathrm{TM}}$}

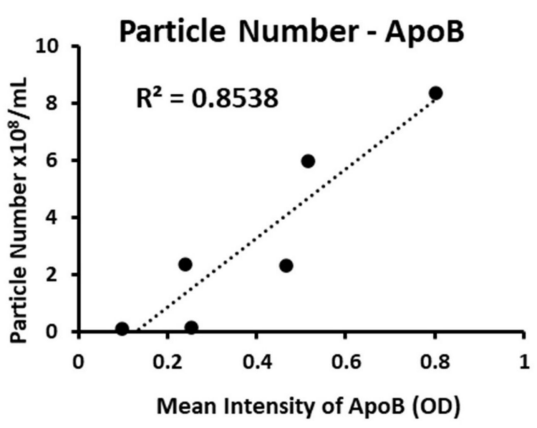

(b)

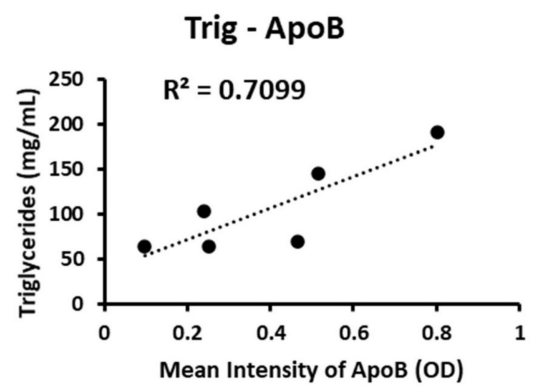

(d)

Trig - Particle Number

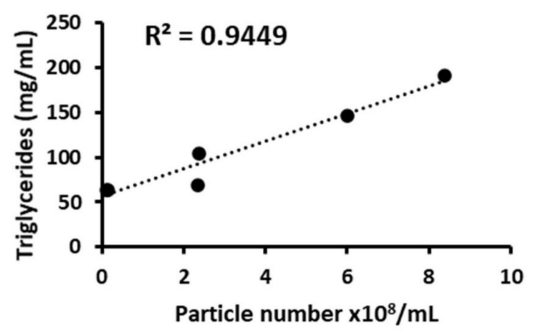

(f)

Figure 4. Lipoprotein contamination in small extracellular vesicles (sEV) preparations purified by differential ultracentrifugation (left column) and Exo-spin ${ }^{\mathrm{TM}}$ (right column). Graphs (a,b) present the correlation between isolated particle numbers (y axis) and mean Apolipoprotein B (ApoB) intensity ( $x$ axis) measured by ELISA in sEV preparations. Graphs $(\mathbf{c}, \mathbf{d})$ show the correlation between triglyceride concentrations (y axis) measured in serum prior to isolation and ApoB intensity ( $x$ axis) in sEV preparations. Graphs (e,f) show the correlation between triglyceride concentrations (y axis) measured in serum prior to isolation and particle number in SEV preparations ( $x$ axis). Statistical analysis of regression was performed in Microsoft Excel with the Data Analysis Tool. 


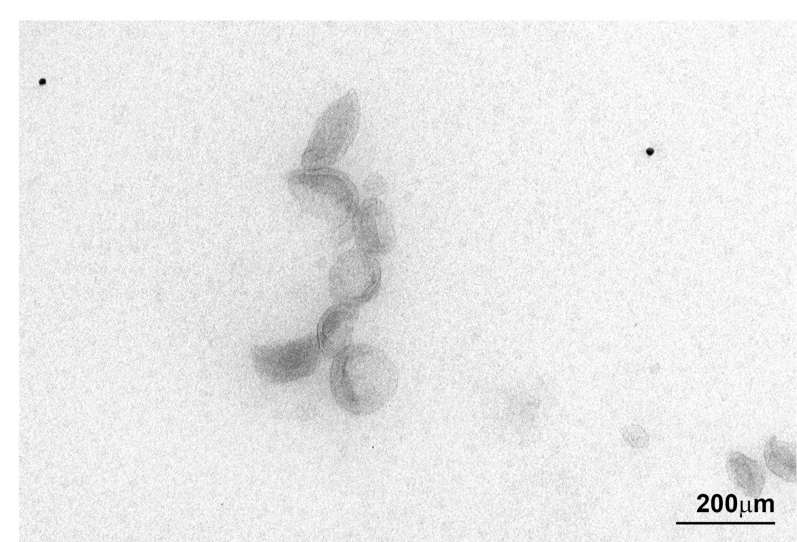

(a)

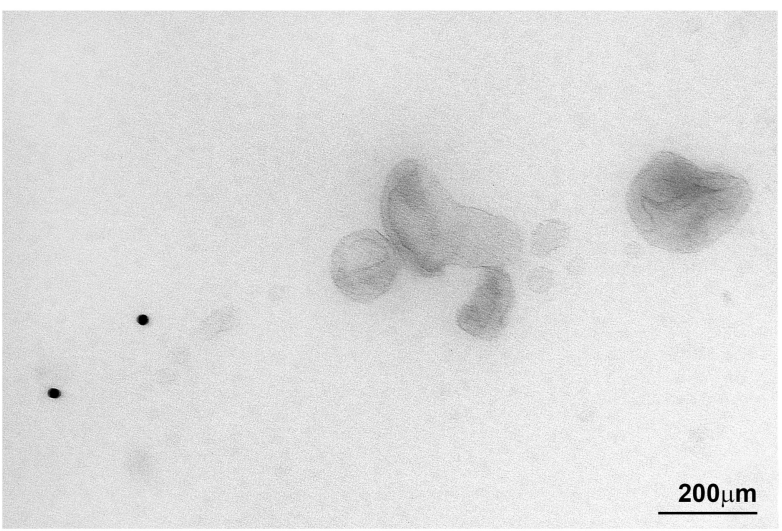

(c)

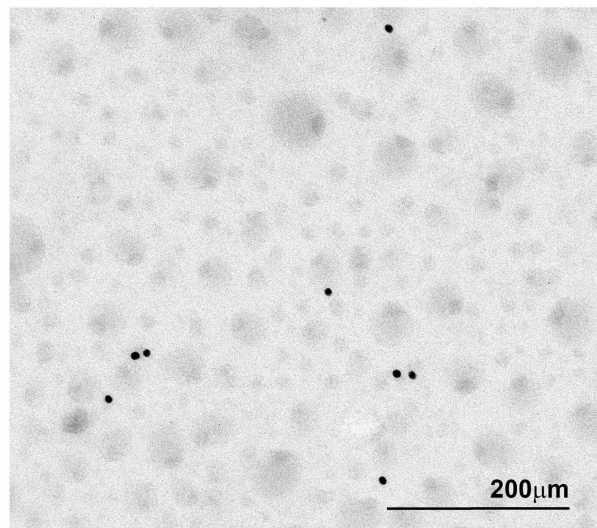

(b)

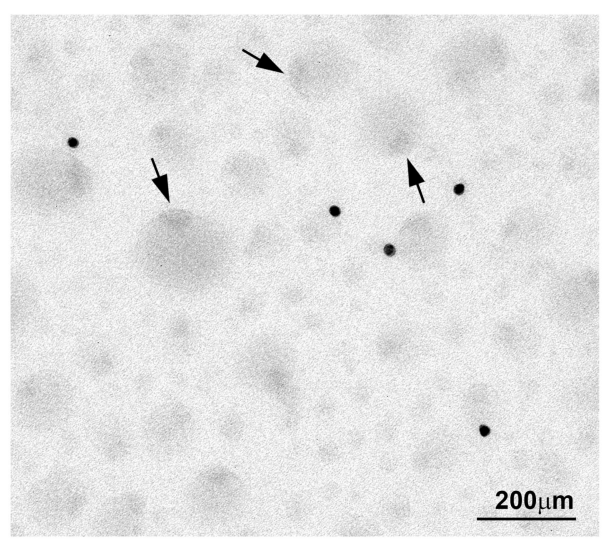

(d)

Figure 5. Transmission electron microscopy images of small extracellular vesicle preparations purified by differential ultracentrifugation (DUC) $(\mathbf{a}, \mathbf{c})$ or Exo-spin ${ }^{\mathrm{TM}}(\mathbf{b}, \mathbf{d})$ that were incubated with an antibody specific for Apolipoprotein B (ApoB). Antibody binding was detected using a secondary antibody conjugated to $15 \mathrm{~nm}$ gold particles. The arrows indicate vesicles that resemble lipid droplets with a triglyceride pocket.

\subsection{MACSPlex Analysis of Membrane Proteins on sEV Purified by DUC and Exo-spin ${ }^{\mathrm{TM}}$}

We used bead-based multiplex EV analysis by flow cytometry (MACSPlex Exosome Kit, human, Miltenyi Biotec) to characterize proteins expressed on the surface of the serumderived sEV. The kit consists of a set of 39 hard-dyed capture bead populations, each of them coated with different monoclonal antibodies specific for one of 37 proteins reported to be expressed on the exosome surface, together with antibodies of two control proteins. The antibody-coated beads capture sEV expressing the relevant molecule, which can then be quantified by flow cytometry after tagging with a cocktail of labelled antibodies to CD9, CD63 and CD81. Paired samples of DUC and Exo-spin ${ }^{\mathrm{TM}}$ purified sEV from six donors were analyzed, and as expected, median allophycocyanin (APC) fluorescence intensities from the DUC samples were consistently higher (Figure 6a shows means $(n=6)$ of median APC fluorescence for each marker and individual median APC fluorescence intensities are shown in the Supplementary Table S2). In the DUC preparations, we obtained median APC fluorescence intensities above the limit of detection for 21 of the 37-exosome proteins, and 10 in the Exo-spin ${ }^{\mathrm{TM}}$ samples (Table 1). Next, we calculated the mean from all median APC fluorescence intensities for the proteins as an estimate of the abundance of exosomes in the preparation, and these were again significantly higher in the DUC preparations (Figure 6a): DUC—7124.80 $\pm 12,203.11$; Exo-spin ${ }^{\mathrm{TM}}-1462.42 \pm 2451.13$ ( $p=0.00012$, Wilcoxon signed rank test). These summary data are mirrored by the median APC fluorescence intensities of specific exosomal markers CD9, CD63 and CD83, which were also uniformly higher in the DUC preparations (Figure 6b-i): CD9-29,015.8 $\pm 16,072.4$ versus $5194.5 \pm 4542.6$ 
$(p=0.031$, Wilcoxon signed rank test); CD63-28,660.0 $\pm 12,303.0$ versus $11,307.8 \pm 8743.4$

$(p=0.031$, Wilcoxon signed rank test); and CD81-5905.2 \pm 3686.9 versus $3763.8 \pm 3586.7$

$(p=0.16$, Wilcoxon signed rank test). However, 20-fold more serum was used to purify sEV by DUC than by for the Exo-spin ${ }^{\mathrm{TM}}$ samples, which were also re-suspended at twice the dilution. Correcting for these differences showed that Exo-spin ${ }^{\mathrm{TM}}$ was at least as efficient as DUC at isolating sEV from human sera, and probably more so.

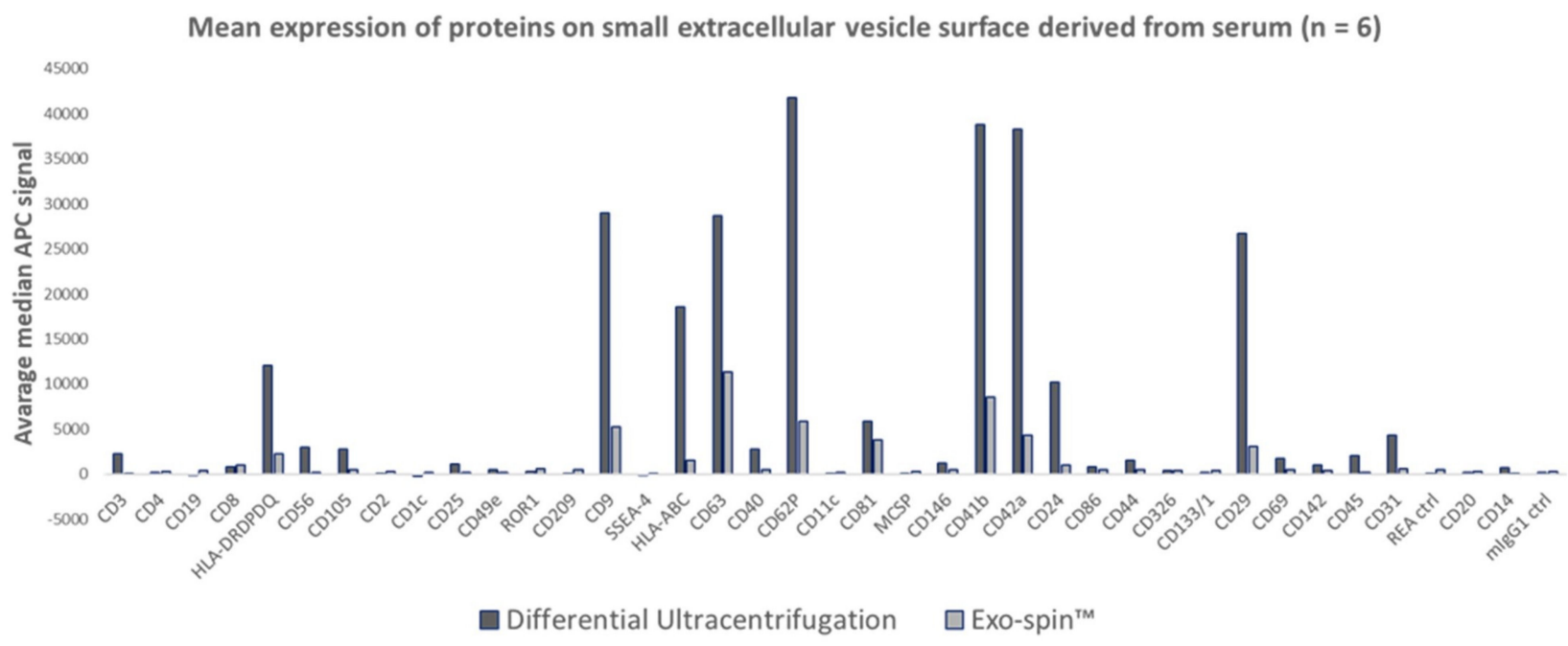

(a)

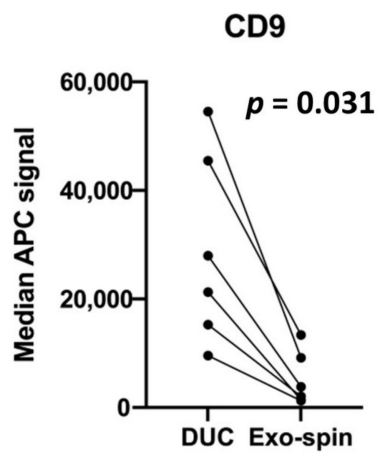

(b)

HLA-ABC

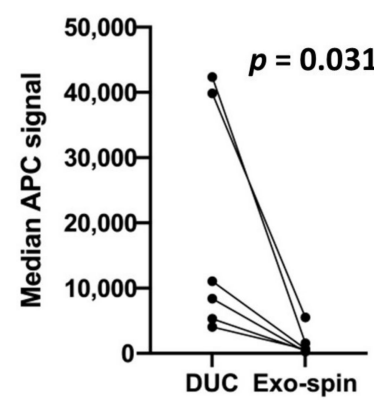

(f)

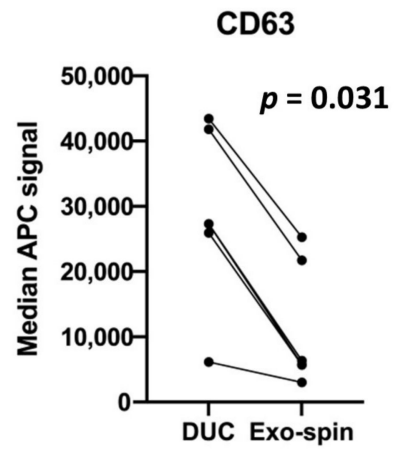

(c)

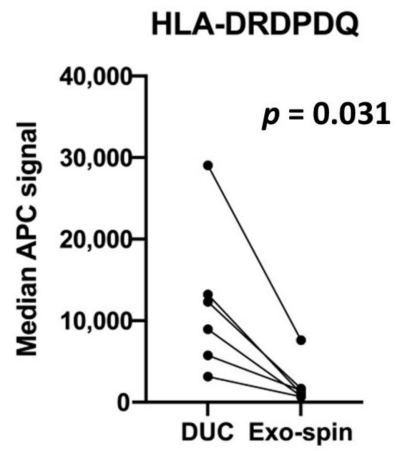

(g)
CD81

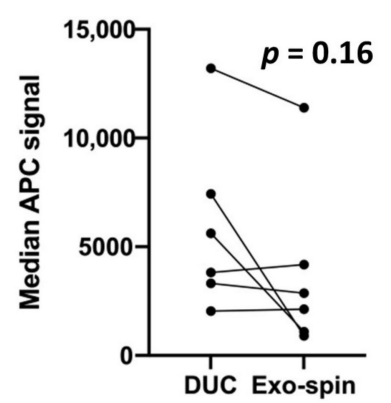

(d)

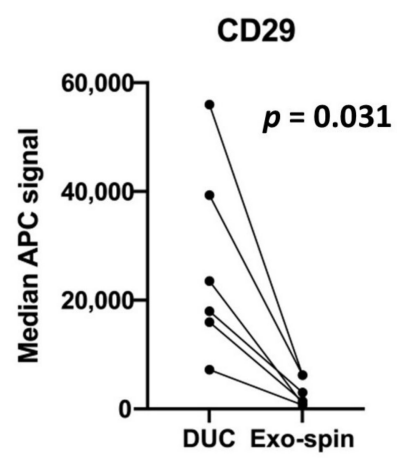

(h)

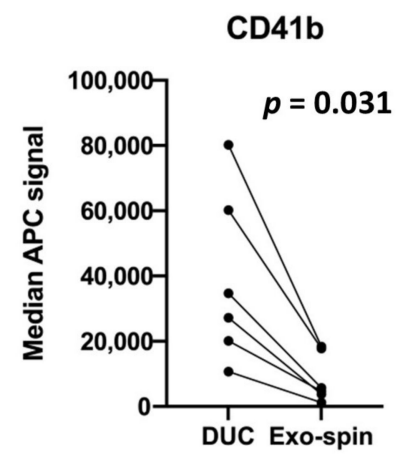

(e)

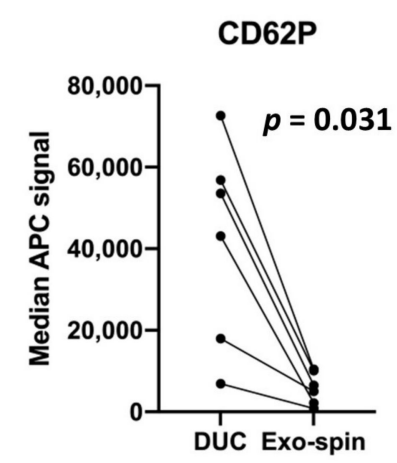

(i)

Figure 6. MACSPlex assay of small extracellular vesicle (sEV) preparations purified by Exo-spin ${ }^{\mathrm{TM}}$ and differential ultracentrifugation (DUC). (a) presents mean of median APC fluorescence intensities shown on axis y $(n=6)$ for all markers included in the kit (x axis). (b-i) show median APC fluorescence intensities (y axis) from sEV preparations purified from 6 donors (each dot $=1$ donor) and paired with the corresponding samples obtained by the other method ( $x$ axis). Statistical analysis was performed in $\mathrm{R}$ with Wilcoxon signed rank test for paired samples. 
Table 1. Mean median allophycocyanin (APC) fluorescence intensities of markers detected by MACSPlex kit in preparations of small extracellular vesicles purified by differential ultracentrifugation (DUC) and Exo-spin ${ }^{\mathrm{TM}}$ (paired samples from 6 donors). The negative controls are marked in grey color. The markers were regarded as present when their median APC fluorescence intensities were above 1000 procedure defined units.

\begin{tabular}{|c|c|c|}
\hline Marker & DUC & Exo-spin $^{\mathrm{TM}}$ \\
\hline CD62P & 41,826 & 5839 \\
\hline CD41b & 38,839 & 8594 \\
\hline CD42a & 38,260 & 4270 \\
\hline CD9 & 29,016 & 5195 \\
\hline CD63 & 28,660 & 11,308 \\
\hline CD29 & 26,669 & 3089 \\
\hline HLA-ABC & 18,512 & 1518 \\
\hline HLA-DRDPDQ & 12,069 & 2206 \\
\hline CD24 & 10,192 & 957 \\
\hline CD81 & 5905 & 3764 \\
\hline CD31 & 4360 & 606 \\
\hline CD56 & 2969 & 153 \\
\hline CD105 & 2781 & 512 \\
\hline CD40 & 2759 & 451 \\
\hline CD3 & 2291 & 33 \\
\hline CD45 & 2090 & 191 \\
\hline CD69 & 1721 & 482 \\
\hline CD44 & 1522 & 525 \\
\hline CD146 & 1234 & 502 \\
\hline CD25 & 1121 & 221 \\
\hline CD142 & 1011 & 417 \\
\hline CD86 & 788 & 481 \\
\hline CD8 & 765 & 1052 \\
\hline CD14 & 679 & 75 \\
\hline CD49e & 466 & 155 \\
\hline CD326 & 369 & 360 \\
\hline ROR1 & 291 & 556 \\
\hline mIgG1 ctrl & 210 & 281 \\
\hline CD133/1 & 156 & 378 \\
\hline CD4 & 139 & 320 \\
\hline CD20 & 132 & 323 \\
\hline REA ctrl & 128 & 504 \\
\hline CD2 & 85 & 265 \\
\hline MCSP & 55 & 258 \\
\hline CD209 & 36 & 451 \\
\hline CD11c & 0 & 233 \\
\hline CD19 & -16 & 352 \\
\hline SSEA-4 & -22 & 4 \\
\hline CD1c & -198 & 161 \\
\hline
\end{tabular}

The MACSPlex assays also allowed us to characterize the populations of SEV in DUC and Exo-spin ${ }^{\mathrm{TM}}$ samples by comparing the median APC fluorescence intensities of all 37 exosome surface proteins. The previously described heat map (Figure 6) shows striking similarities between the DUC and Exo-spin ${ }^{\mathrm{TM}}$ samples, which we quantified by both correlating the mean APC signals for all proteins (multiple $\mathrm{R}=0.85, p=4.03 \times 10^{-12}$ ), and by correlating the paired sEV samples from each of the donors (Multiple $\mathrm{R}=0.81 \pm 0.10$ (range: 0.63-0.94)). The correlated abundance of individual markers is shown in Figure 7. Taken together, the MACSPlex data demonstrate that DUC and Exo-spin ${ }^{\mathrm{TM}}$ purify identical populations of sEV from a given serum sample. They also provide the initial data about the cellular origins of the $\mathrm{sEV}$, since there were recurrent signals from multiple donors suggesting leukocytes (CD24, CD40, CD45, and CD56), endothelium (CD31, CD62P, and $\mathrm{CD} 105)$, and platelets (CD41b and CD42a) were consistent sources. Coincidentally, the data 
also show that the co-purified lipoproteins do not interfere with antibody-based strategies for characterizing proteins expressed on the sEV membrane.

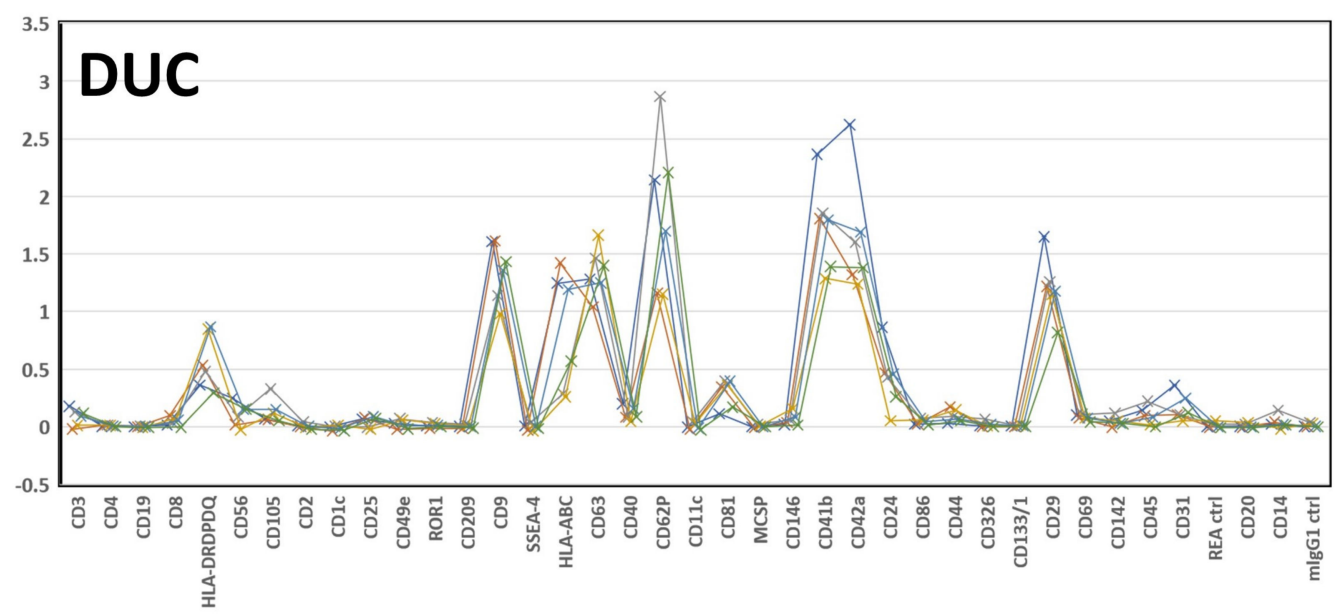

(a)

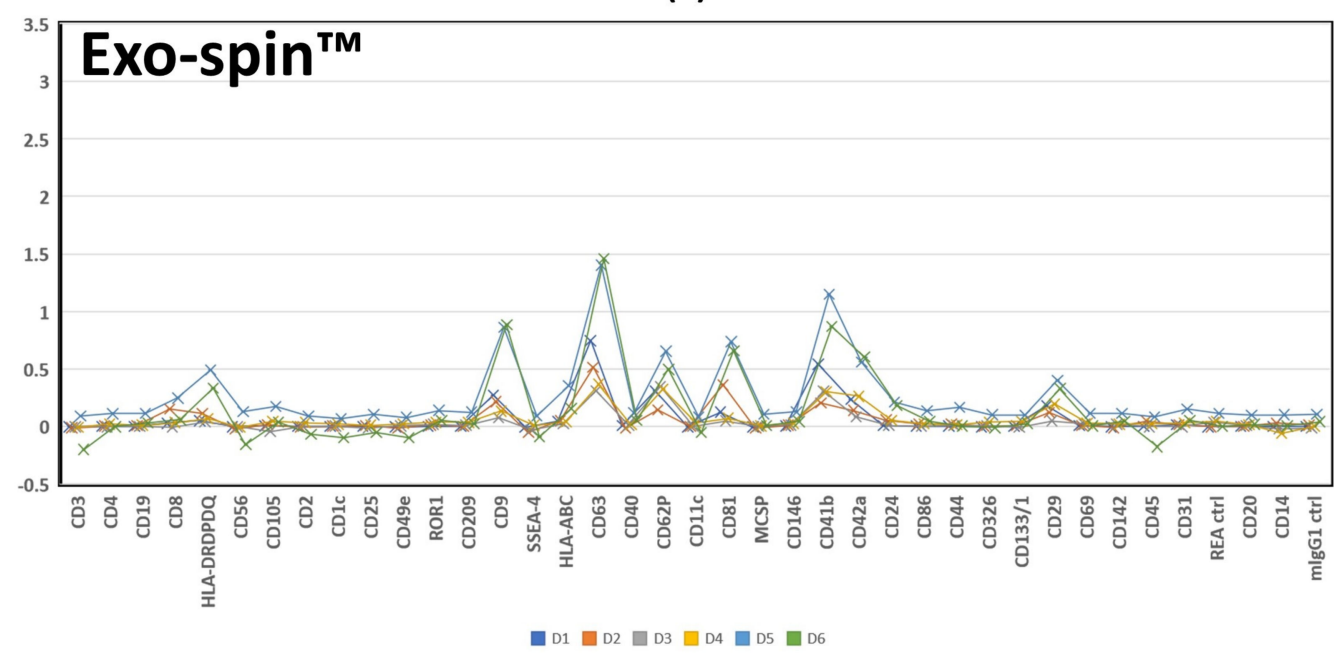

(b)

Figure 7. Correlation between 39 proteins median allophycocyanin (APC) fluorescence intensities acquired by bead-based multiplex EV analysis (MACSPlex Exosome Kit, human, Miltenyi Biotec) of small extracellular vesicle sEV) preparations purified by differential ultracentrifugation (a) (DUC) and Exo-spin ${ }^{\mathrm{TM}}$ (b). The median APC fluorescence intensities of 39 proteins shown on axis y were normalized to the mean of CD9, CD63 and CD81 median APC fluorescence intensity of each sEV sample. Each colored line corresponds to one donor and the samples between methods are paired. The specific markers are shown on axis $\mathrm{x}$.

\section{Discussion}

The molecules expressed in the limiting membranes of circulating exosomes, together with cargo within their lumens, bear the imprint of the cell and tissue in which they originated, and whether it is stressed or injured $[5,26,27]$. These are highly attractive properties for non-invasive probes for human disease diagnosis and monitoring, but exploitation of exosomes as biomarkers that have been hindered by uncertainties about how best to isolate them from human blood. Here we report a detailed comparison of their isolation from human serum by DUC and the much simpler method of Exo-spin ${ }^{\mathrm{TM}}$ columns (Table 1). Our results show that: (i) both techniques efficiently purify similar populations of sEV from human serum, but larger numbers can be obtained by DUC; (ii) Exo-spin ${ }^{\mathrm{TM}}$ columns co-purify copious lipoprotein particles together with the $\mathrm{sEV}$, and this invalidates the use of NTA to measure sEV yield, which can only be inferred from the abundance of 
exosome specific proteins, such as CD9, CD63 and CD81; and (iii) despite obvious donorto-donor variation in abundance, MACSPlex analysis of sEV surface proteins uniformly demonstrate strong signals indicating their leukocyte, platelet, and endothelial origins. Our results provide essential information about purification and quality control of sEV from human serum and raise questions about the choice of method for particular studies.

Differential ultracentrifugation is a well established method for purifying sEV that can be applied to relatively large volumes of serum, but it is too laborious to be used for routine clinical patient monitoring, though not for research studies. By contrast, Exo-spin ${ }^{\mathrm{TM}}$ is simple enough for routine clinical use, but can only be applied to small volumes of serum (100 $\mu \mathrm{L}$ for the Exo-spin ${ }^{\mathrm{TM}}$ used in this study) [28]. Our data confirm the effectiveness of Exo-spin ${ }^{\mathrm{TM}}$ columns for isolating $\mathrm{sEV}$ from human sera and show that, based on the abundance of CD9, CD63 and CD81, they are at least as efficient as DUC, and probably more so, when the results had corrections had been made for the different volumes of serum used (100 $\mu \mathrm{L}$ and $2 \mathrm{~mL}$ respectively). Nevertheless, the relatively small volumes of serum that can be applied to Exo-spin ${ }^{\mathrm{TM}}$ columns limits analytical studies that can be applied to the purified sEV. This was highlighted by the MACSPlex analysis, which identified 21 different proteins in DUC purified sEV samples, but only 10 in those purified by Exo$\operatorname{spin}^{\mathrm{TM}}$; the remaining 11 were below the limit of detection in the assay. Nevertheless, the similarity of the abundance profiles for the proteins identified by MACSPlex confirms that both techniques isolate identical populations of sEV. The failure to identify low abundance proteins of Exo-spin ${ }^{\mathrm{TM}}$ samples could be addressed by using a larger column, such as the Exo-spin ${ }^{\mathrm{TM}}$ EXO4, with a maximum volume of but the advantage of DUC is that there is no limit to volumes of serum that can be sampled, and thus it is possible to isolate as many $\mathrm{sEV}$ as is required.

Co-purification of other blood components with the sEV is a challenge for all the methods, and contamination with lipoproteins, and in particular VLDL and small chylomicrons, has previously been reported [20,29-31]. However, the lower density of lipids separates most of them from sEV during DUC, but their overlapping size ranges with sEV and their abundance in serum $\left(1 \times 10^{12} / \mathrm{mL}\right)$ [32] proved to be a major issue in the Exo-spin ${ }^{\mathrm{TM}}$ separations. This was highlighted by NTA, which showed that Exo-spin ${ }^{\mathrm{TM}}$ samples contained around 500 times more particles per unit volume of blood than the DUC preparations, despite their significantly lower concentrations of exosome specific proteins. The first evidence that high particle counts were co-purified lipoproteins came from the close correlation between particle number and ApoB concentrations in Exo-spin ${ }^{\mathrm{TM}}$ samples, and with serum triglycerides. We suspect that VLDL is the main source of the lipid particles, since they are normally between 30 and $90 \mathrm{~nm}$ in diameter [33]. The similarities between the ultrastructural appearances of IDL [34], VLDL [35], HDL [36] and the particles in Exo-spin ${ }^{\mathrm{TM}}$ samples strongly support this interpretation, which is highlighted by the presence of lipid droplets with their characteristic triglyceride pockets [25]; immunoEM with antibodies specific for ApoB confirmed the excess of lipoproteins in the Exo-spin ${ }^{\mathrm{TM}}$ samples, even though the density of the particles and the number of gold beads prevented us from directly linking the two. By contrast, most vesicles in the DUC preparations had the typical appearance of exosomes, albeit sometimes in clumps or aggregates, and immunoEM studies showed that many expressed CD9 and CD63, which confirmed their nature; only a very small fraction of vesicles in the Exo-spin ${ }^{\mathrm{TM}}$ preparations expressed these exosome markers. Thus, DUC results in relatively pure population of sEV whose yield can be estimated by NTA or from the abundance of CD9, CD63 and CD81. By contrast, the yield of sEV in Exo-spin ${ }^{\mathrm{TM}}$ preparations can only be estimated by the abundance of exosome specific proteins.

There were marked donor-to-donor variations in the yields of sEV. Blood was always drawn in the morning, but donors were not fasting and we did not control for physical activity, which could affect the concentration of circulating sEV [37]; nevertheless, these are unlikely to completely account for the differences we observed. It is striking that donor related differences in yield were conserved across the various measurement, regardless 
of the purification method, and whether the sEV yield was estimated by CD9, CD63 and CD81 abundance or MACSPlex analysis, or in the case of DUC, by NTA; this strongly favors a biological rather than a technical explanation. The MACSPlex data also provides initial data about the cellular source of the sEV in the serum with multiple recurrent signals suggesting leukocytes (CD24, CD40, CD45, and CD56), endothelium (CD31, CD62P, and CD105), and platelets (CD41b and CD42a) although the latter has been released ex-vivo. Nevertheless, these data provide strong encouragement for studies of sEV as biomarkers of disease activity.

In conclusion, we have shown that $\mathrm{sEV}$ can be purified efficiently from human serum by differential ultracentrifugation (DUC) and size exclusion chromatography (Exo-spin ${ }^{\mathrm{TM}}$ EX05 mini-HD), but that NTA cannot be used to calculate yields with the latter. The main differences between the methods are summarized in Table 2. Our data provide the foundation for the studies in the use of sEV as "liquid biopsies" to study human disease.

Table 2. Summary of small extracellular vesicles properties purified by differential ultracentrifugation (DUC) and Exo$\operatorname{spin}^{\mathrm{TM}}$.

\begin{tabular}{|c|c|c|c|}
\hline Characteristics & DUC & Exo-spin ${ }^{\mathrm{TM}}$ & Comments \\
\hline $\begin{array}{l}\text { Minimal serum volume for } \\
\text { healthy individuals }\end{array}$ & $1 \mathrm{~mL}$ & $100 \mu \mathrm{L}$ & $\begin{array}{c}\text { DUC range: } 1-5 \mathrm{~mL} \\
\text { Exo-spin }{ }^{\mathrm{TM}} \text { range: } x-150 \mu \mathrm{L}\end{array}$ \\
\hline $\begin{array}{l}\text { Final volume of sEV } \\
\text { resuspended in PBS }\end{array}$ & $200 \mu \mathrm{L}$ & $400 \mu \mathrm{L}$ & $\begin{array}{l}\text { Volume of DUC can be adjusted for } \\
\text { individual purposes }\end{array}$ \\
\hline Total protein concentration & $32.4 \pm 7.8 \mu \mathrm{g} / \mathrm{mL}$ & $40.6 \pm 16.8 \mu \mathrm{g} / \mathrm{mL}$ & \\
\hline \multirow{2}{*}{$\begin{array}{l}\text { Recovery (particle } \\
\text { number/mL of serum) }\end{array}$} & \multirow{2}{*}{$1.0 \pm 1.1 \times 10^{8} / \mathrm{mL}$} & \multirow{2}{*}{$497.6 \pm 769.5 \times 10^{8} / \mathrm{mL}$} & $\begin{array}{l}\text { High recovery of particles by Exo-spin is } \\
\text { mostly associated with large numbers of } \\
\text { lipoproteins being co-isolated; }\end{array}$ \\
\hline & & & $\begin{array}{l}\text { Normalisation is essential since the } \\
\text { initial applied volume of serum differs }\end{array}$ \\
\hline $\begin{array}{l}\text { Absolute number of } \\
\text { particles }\end{array}$ & $2.7 \pm 2.2 \times 10^{8}$ & $49.8 \pm 76.9 \times 10^{8}$ & $\begin{array}{l}\text { Absolute number of particles that is } \\
\text { purified from heathy individuals } \\
\text { according to the suited protocol }\end{array}$ \\
\hline Particle size (diameter) $\varnothing$ & $\begin{array}{c}\text { Mean }=148.1 \pm 14.6 \mathrm{~nm} \\
\text { Mode }=121.9 \pm 20.6 \mathrm{~nm} \\
\text { Median }=138.0 \pm 13.7 \mathrm{~nm} \\
\% \text { of particles }<150 \mathrm{~nm}=57.66 \pm \\
8.8 \%\end{array}$ & $\begin{array}{c}\text { Mean }=132.5 \pm 12.1 \mathrm{~nm} \\
\text { Mode }=110.9 \pm 17.2 \mathrm{~nm} \\
\text { Median }=123.1 \pm 13.1 \mathrm{~nm} \\
\% \text { of particles }<150 \mathrm{~nm}=69.6 \pm \\
8.4 \%\end{array}$ & $\begin{array}{l}\text { Large size of particles isolated by DUC } \\
\text { may be associated with aggreagates } \\
\text { induction by gravitational forces }\end{array}$ \\
\hline $\begin{array}{l}\text { Relative mean intensity by } \\
\text { ELISA }\end{array}$ & $\begin{array}{l}\text { CD81: } 0.36 \pm 0.18 \\
\text { CD63: } 0.52 \pm 0.25 \\
\text { CD9: } 0.96 \pm 0.48\end{array}$ & $\begin{array}{l}\text { CD81: } 0.12 \pm 0.08 \\
\text { CD63: } 0.16 \pm 0.07 \\
\text { CD9: } 0.18 \pm 0.07\end{array}$ & $\begin{array}{l}\text { Measured particle concentrations for } \\
\text { these samples were: DUC }=10.54 \times \\
10^{8} / \mathrm{mL} \text {; Exo-spin }{ }^{\mathrm{TM}}=32.25 \times 10^{8} / \mathrm{mL}\end{array}$ \\
\hline $\begin{array}{l}\text { Median APC fluorescence } \\
\text { intensity by MACSPlex }\end{array}$ & $\begin{array}{l}\text { Average: } 7124.8 \pm 12,203.1 \\
\text { CD81: } 5905.2 \pm 3686.9 \\
\text { CD63: } 28,660 \pm 12,303 \\
\text { CD9: } 29,016 \pm 16,072\end{array}$ & $\begin{array}{l}\text { Average: } 1462.4 \pm 2451.1 \\
\text { CD81: } 3763.8 \pm 3586.7 \\
\text { CD63: } 11,308 \pm 8743.4 \\
\text { CD9: } 5194.5 \pm 4542.6\end{array}$ & All samples were paired \\
\hline Morphology & $\begin{array}{l}\text { Singles and aggregates of vesicles, } \\
\text { cup-like shape, approximately } \\
\text { half of vesicle were stained } \\
\text { positively with exosomal markers }\end{array}$ & $\begin{array}{l}\text { Whole image fully layered with } \\
\text { vesicles, various morphologies } \\
\text { that does not resemble exosomes, } \\
\text { only few stained positively with } \\
\text { exosomal markers }\end{array}$ & - \\
\hline \multirow[t]{2}{*}{ Co-purification } & Low lipoprotein contamination & High lipoprotein contamination & $\begin{array}{l}\text { Most of the particles isolated by } \\
\text { Exo-spin }{ }^{\mathrm{TM}} \text { from non-fasted patients } \\
\text { were assigned as lipoproteins; }\end{array}$ \\
\hline & $\begin{array}{l}\text { Albumin contamination } \\
\text { approximately } 12.85 \%\end{array}$ & $\begin{array}{l}\text { Non-detectable albumin } \\
\text { contamination }\end{array}$ & $\begin{array}{l}\text { Albumin contamination was evaluated } \\
\text { by silver staining. }\end{array}$ \\
\hline
\end{tabular}

Abbreviations: APC—allophycocyanin; DUC—differential ultracentrifugation; ELISA—enzyme-linked immunoassay; sEV—small extracellular vesicles. 


\section{Materials and Methods}

\subsection{Blood Samples}

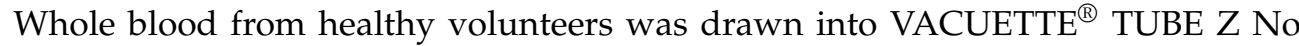
Additive (Greiner Bio-One, Austria), and left to clot for 20-30 min at room temperature before being centrifuged $2500 \times g$ for $15 \mathrm{~min}$ at room temperature.

\subsection{Isolation of sEV by Differential Ultracentrifugation (DUC)}

Samples of $2 \mathrm{~mL}$ of serum were diluted in $31 \mathrm{~mL}$ of cold PBS in order reduce the viscosity and albumin concentration before being subjected to differential ultracentrifugation (Allegra X-15R, Beckman Coulter, Brea, CA, USA; Ultracentrifuge CP100NX, Hitachi, Tokyo, Japan). Large vesicles were removed by ultracentrifugation at $30,000 \times g$ for $2 \mathrm{~h}$ at $4{ }^{\circ} \mathrm{C}$. The supernatant was collected and sEV were pelleted at $100,000 \times g$ for $2 \mathrm{~h}, 4^{\circ} \mathrm{C}$. The supernatant was discarded and the pellet was resuspended in $33 \mathrm{~mL}$ of cold PBS before re-pelleting with a second ultracentrifugation step $\left(100,000 \times g ; 2 \mathrm{~h} ; 4^{\circ} \mathrm{C}\right)$ to wash the sEV. After discarding the supernatant, the sEV-containing pellet was gently resuspended and transferred to an Eppendorf tube, in which the volume was adjusted to a total of $200 \mu \mathrm{L}$ by adding cold PBS. Initial experiments established DUC could be used to harvest sEV from between 0.5 to $10 \mathrm{~mL}$ serum (optimal volume 1 to $5 \mathrm{~mL}$ ) (Supplementary Figure S8); we used a standard volume of $2 \mathrm{~mL}$ for all the experiments reported here. Additionally, we determined the repeatability of this isolation method described by the number of recovered particles, size and distribution (Supplementary Figure S9).

\subsection{Isolation of sEV by Exo-spin ${ }^{\mathrm{TM}}$ Mini-HD Column EX05}

Exo-spin ${ }^{\mathrm{TM}}$ mini-HD column EX05 (Cell Guidance Systems, Cambridge, UK) - in the text generally referred to as Exo-spin ${ }^{\mathrm{TM}}$ — were used to isolate sEV from $100 \mu \mathrm{L}$ of serum and resuspended in $400 \mu \mathrm{L}$ of PBS, according to the manufacturer's instructions. Briefly, $100 \mu \mathrm{L}$ of sera were centrifuged for $30 \mathrm{~min}, 14,000 \times g$ and loaded onto the columns after equilibrating them with $3 \times 2.5 \mathrm{~mL}$ PBS. After washing with $900 \mu \mathrm{L}$ of PBS, the sEV fraction was eluted into an Eppendorf tube with $400 \mu \mathrm{L}$ of PBS.

\subsection{Nanoparticle Tracking Analysis}

Nanoparticle tracking analysis captures videos of microscopic images of light scattering induced by Brownian motion of vesicles and protein aggregates. The videos are analyzed by the NTA software, which calculates the size of single particles and their concentration [32,38,39]. We used a Zetaview ${ }^{\circledR}$ Basic NTA PMX 120 (Particle Metrix, Inning am Ammersee, Germany) system. All samples were freshly diluted in PBS, which was set as a background noise within these experiments. We evaluated the standard settings that were kept throughout the whole experiment: sensitivity $=68$; shutter $=65$; temperature $=24{ }^{\circ} \mathrm{C}$. The dilution factor (DF) was changed for samples depending on an estimated particle concentration in samples (for DUC, DF $=25-150$; for Exo-spin ${ }^{\mathrm{TM}}$, DF $=150-1500$ ) in order to fit the concentration range: $10^{5}-10^{9}$ particles $/ \mathrm{mL}$. For each measurement, three cycles were performed by scanning 11 cell positions each and capturing 30 frames per position. The images were analyzed with FlowJo_v10.6.1 (FlowJo LLC, Ashland, OR, USA) and R version 3.6.3 (R Core Team) for Windows.

\subsection{Primary and Secondary Antibodies}

The primary antibodies used in transmission electron microscopy and Western blotting and ELISA were as follows: mouse anti-CD63 (10628D; Invitrogen, Waltham, MA, USA); mouse-anti CD9 (AHS0902, Invitrogen, Waltham, MA, USA); and mouse anti-ApoB100/48 (3715-3-250, MabTech, Nacka Strand, Sweden). Antibodies anti-CD81 (10630D, Invitrogen, Waltham, MA, USA) and anti-Calnexin (C5C9) (\#2679, Cell Signaling Technology, Danvers, MA, USA) were only used on ELISA.

The secondary antibody used in transmission electron microscopy (TEM) was goldlabelled (EM:GAM15, goat anti-mouse 15 nm immunogold-conjugate; BBI Solution, Crum- 
lin, UK). For Western blotting, we used fluorescently labelled secondary antibodies: goat anti mouse IRDye ${ }^{\circledR} 800$ or goat anti mouse IRDye ${ }^{\circledR} 680$ or goat anti rabbit IRDye ${ }^{\circledR} 680$ or goat anti rabbit IRDye ${ }^{\circledR} 800$ (LI-COR Biosciences, Lincoln, NE, USA). The ELISA secondary antibodies were conjugated with horseradish peroxidase (HRP) as follows: Goat anti rabbit- HRP (111-036-047, Jackson Immunoresearch, West Grove, PA, USA); or Rabbit anti mouse-HRP (JZM035046_Fa, Ancell Immunology Research, Bayport, MN, USA).

\subsection{Transmission Electron Microscopy and Immuno-Electron Microscopy}

Transmission electron microscopy was performed, as described by Thery et al. [16]. Briefly, the small extracellular vesicle (sEV) preparations were fixed in $2 \%$ PFA and a $5 \mu \mathrm{L}$ drop was placed on formvar-coated 200 mesh Ni-grids (S162N; Agar Scientific Ltd., Standsted, UK). CD63 and CD9 was detected in sEV by immuno-electron microscopy after incubating the grids on drops of the antibody dilution in 1\% Eggalbumin overnight in a humid chamber at $4{ }^{\circ} \mathrm{C}$ with mouse anti-CD63 at a 1:40 dilution, mouse- anti CD9 at a 1:20 dilution, or mouse anti-ApoB100/48 at a 1:10 dilution. Binding of antibodies was detected with a gold-labelled secondary antibody goat anti mouse-diluted 1:50. Washing steps between antibody incubation were performed with PBS followed by post-fixation with $1 \%$ glutaraldehyde, incubation in a solution of neutral uranyl oxalate and covering with a polyvinylalcohol-film. After air-drying, the grids were examined in a TEM JEOL 1400 PLUS (JEOL, Tokyo, Japan) at 60 kV. Pictures were taken in Radius-software with Quemesa_Camera (Fa. Olympus, Tokyo, Japan).

\subsection{SDS-PAGE and Western Blotting}

Small extracellular vesicle preparations were purified by differential ultracentrifugation and Exo-spin ${ }^{\mathrm{TM}} .30 \mu \mathrm{L}$ of each sEV sample was mixed with $10 \mu \mathrm{L} 4 \times$ Protein Loading Buffer (LI-COR, Biosciences, Lincoln, NE, USA) and incubated at $95{ }^{\circ} \mathrm{C}$ for $5 \mathrm{~min}$. The samples were separated on either $10 \%$ or $4-20 \%$ gradient SDS-PAGE non-reducing conditions. In some conditions, dilutions of human serum, lysates of THP-1 cells or purified albumin were added as protein loading controls. Cells were lysed in RIPA buffer (150 mM NaCl; 5mM EDTA, pH 8.0; 50 mM TRIS, pH 8.0; 1\% Triton X-100; 0.5\% sodium deoxycholate; $0.1 \%$ SDS) by sonication.

Silver staining of SDS gels was performed with PlusOne ${ }^{\mathrm{TM}}$ Silver staining kit, Protein (GE Healthcare Bio-Sciences AB, Stockholm, Sweden) according to the manufacturer's instructions. For Western blot analysis, gels were transferred onto a nitrocellulose membrane (GE Healthcare, Chicago, IL, USA). The membranes were blocked for 1h in RT with Intercept $^{\circledR}$ (TBS) Blocking Buffer (LI-COR, Biosciences, Lincoln, NE, USA) or Odyssey ${ }^{\circledR}$ (PBS) Blocking Buffer (LI-COR, Biosciences, Lincoln, NE, USA). Incubation with primary antibodies to either CD63 or CD9 was performed under constant shaking for $1 \mathrm{~h}$ at RT or overnight at $4{ }^{\circ} \mathrm{C}$. Next, the membranes were washed with TBST (TBS $+0.1 \%$ Tween20) and incubation with the appropriate secondary antibody was performed for $1 \mathrm{~h}$ at RT (goat anti mouse or goat anti rabbit). Three washing steps between and after each antibody incubation were followed by visualization using an Odyssey Infrared Imager (LI-COR, Biosciences, Lincoln, NE, USA). Analysis was performed with ImageJ Studio Lite Version 5.2. (Rasband, W.S., ImageJ, U.S. National Institutes of Health, Bethesda, MD, USA)

\subsection{ELISA}

ELISA was performed using polystyrene 96-well plates (Nunc ${ }^{\mathrm{TM}}$, Roskilde, Denmark) with the following antibodies: anti-CD63; anti-CD9; anti-CD81; anti-Calnexin (C5C9); anti-ApoB100/48. Purified sEV were mixed with coating buffer (0.05 M Carbonate buffer; $\mathrm{pH} 9,6)$ at a ratio of $1: 4$ and incubated in the plates for $2 \mathrm{~h}$ at $\mathrm{RT}$ or overnight at $4{ }^{\circ} \mathrm{C}$ on a shaker. After washing the wells 3 times with TBST (TBS, 0.05\% Tween20) for $5 \mathrm{~min}$, blocking was performed with TBS containing 1\% BSA for $2 \mathrm{~h}$ at RT. Primary antibodies diluted in blocking buffer were applied and incubated for $2 \mathrm{~h}$ at RT or overnight at $4{ }^{\circ} \mathrm{C}$ on a shaker. After 3 washes the appropriate secondary antibodies conjugated to HRP were 
applied to the plates followed by $2 \mathrm{~h}$ incubation at RT on a shaker. Antibody binding was visualized by obtaining 2,3-diaminophenazine and color reaction was stopped with $8 \mathrm{~N} \mathrm{H}_{2} \mathrm{SO}_{4}$. The absorbance was measured at $490 \mathrm{~nm}$ and $630 \mathrm{~nm}$ on a plate reader. The blanks were filled with only development buffer and stop buffer. As positive controls we used dilution of human serum and THP-1 cell lysates. As negative controls on the coated plated we applied only secondary antibodies (omitted primary antibody incubation) and only primary antibodies on a coated plate (omitted secondary antibody incubation). The intensity was calculated by subtracting 490 blank by 630 blank (OD), and we evaluated the mean intensity from 3 replicates for each sample-in the figures referred to as "Mean Intensity (OD)".

\subsection{MACPlex Human Exosome Pan Kit}

The analysis was performed using the MACSPlex Exosome Kit, human (Miltenyi Biotec, Bergisch-Gladbach, Germany) according to the manufacturer's instructions. Briefly, small extracellular vesicle preparations $(120 \mu \mathrm{L})$ were incubated overnight with MACSPlex Exosome Capture Beads ( $15 \mu \mathrm{L}$; containing 39 different antibody-coated bead subsets) on an orbital shaker (450 rpm) at RT without access to light. Next, samples were washed by adding $500 \mu \mathrm{L}$ of MACSPlex buffer into each tube and centrifuged at RT for $5 \mathrm{~min} 3000 \times g$. The supernatant was discarded, and the pelleted beads were incubated with MACSPlex Exosome Detection Reagent (APC-conjugated anti-CD63, anti-CD9, anti-CD81) for $1 \mathrm{~h}$ at RT on a shaker (450 rpm). After this, beads were washed twice with MACSPlex buffer and fluorescence intensities for FITC, PE and APC were acquired by the BD FACSCanto II Cell Analyzer (BD Biosciences, Franklin Lakes, NJ, USA), resulting in approximately 5000-20,000 single bead events being recorded per sample. Analysis was performed in FlowJo_v10.7.1. by separating the bead populations according to their fluorescence properties in the PE and FITC channel. Median APC fluorescence was used to quantify the exosomes bound to the beads.

\subsection{Micro BCA ${ }^{\mathrm{TM}}$ Protein Assay Kit}

We determined the total protein concentration in the small extracellular vesicle preparations; we used the Micro BCA ${ }^{\mathrm{TM}}$ Protein Assay Kit $(23,235$, Thermofisher Scientific, Waltham, MA, USA) following the user guide for the microplate procedure. The selected samples were measured in duplicate and in one or two dilutions, depending on the volumes available.

\subsection{Statistical Analysis}

Statistical analysis was performed with $\mathrm{R}$ version 3.6.3 for Windows ( $\mathrm{R}$ Core Team (2020). R: A language and environment for statistical computing. R Foundation for Statistical Computing, Vienna, Austria. URL https:/ /www.R-project.org/and Microsoft Excel (Redmond, WA, USA). We applied Wilcoxon signed rank test and Wilcoxon rank sum test. The graphs were performed in Graphpad Prism 9.0.0 (GraphPad Software, San Diego, CA, USA).

Supplementary Materials: The Supplementary Materials are available online at https:/ / www.mdpi. com/article/10.3390/ijms22094653/s1.

Author Contributions: Conceptualization, M.S.M., A.J.R. and R.K.; methodology, M.S.M., H.S. and S.M.S.; software, M.S.M. and C.A.; analysis, M.S.M.; investigation, M.S.M., A.J.R. and R.K.; writingoriginal draft preparation, M.M.; writing-review and editing, A.R., R.K. and C.A.; visualization, M.S.M. and C.A.; supervision, A.J.R. and R.K.; funding acquisition, R.K. All authors have read and agreed to the published version of the manuscript. 
Funding: This project has received funding from the European Union's Horizon 2020 research and innovation programme under the Marie Skłodowska-Curie grant agreement No. 813545. The information and views set out in this article are those of the authors and do not necessarily reflect the official opinion of the European Union. Neither the European Union institutions and bodies nor any person acting on their behalf may be held responsible for the use which may be made of the information contained therein.

Institutional Review Board Statement: The study was conducted according to the guidelines of the Declaration of Helsinki, and approved by the Medical University Vienna Ethics Committee (ECS 1089/2012 and IRB 1945/2018).

Informed Consent Statement: Informed consent was obtained from all subjects involved in the study.

Acknowledgments: This project has received funding from the European Union's Horizon 2020 research and innovation programme under the Marie Skłodowska-Curie grant agreement No. 813545. The authors acknowledge the Core Facilities of the Medical University of Vienna, a member of Vienna Life-Science Instruments (VLSI). We thank Anton Jäger from dept. of Pathology at Medical University of Vienna for help with illustrations.

Conflicts of Interest: The authors declare no conflict of interest.

$\begin{array}{ll}\text { Abbreviations } \\ \text { APC } & \text { Allophycocyanin } \\ \text { BCA } & \text { Bicinchoninic acid } \\ \text { BSA } & \text { Bovine serum albumin } \\ \text { DF } & \text { Dilution Factor } \\ \text { DUC } & \text { Differential ultracentrifugation } \\ \text { EDTA } & \text { Ethylenediaminetetraacetic acid } \\ \text { ELISA } & \text { Enzyme-linked immunoassay } \\ \text { EV } & \text { Extracellular vesicles } \\ \text { HDL } & \text { High-density lipoproteins } \\ \text { HRP } & \text { Horseradish peroxidase } \\ \text { LDL } & \text { Low-density lipoproteins } \\ \text { MHC } & \text { Major histocompatibility complex } \\ \text { NTA } & \text { Nanoparticle tracking analysis } \\ \text { OPD } & \text { O-Phenylenediamine dihydrochloride } \\ \text { RT } & \text { Room temperature } \\ \text { sEV } & \text { Small extracellular vesicles } \\ \text { TBS } & \text { Tris-buffered saline } \\ \text { TBST } & \text { Tris-buffered saline + Tween20 } \\ \text { TEM } & \text { Transmission electron microscopy } \\ \text { TRIS } & \text { Trisaminomethane }\end{array}$

\section{References}

1. Doyle, L.; Wang, M. Overview of Extracellular Vesicles, Their Origin, Composition, Purpose, and Methods for Exosome Isolation and Analysis. Cells 2019, 8, 727. [CrossRef]

2. Bebelman, M.P.; Smit, M.J.; Pegtel, D.M.; Baglio, S.R. Biogenesis and function of extracellular vesicles in cancer. Pharmacol. Ther. 2018, 188, 1-11. [CrossRef] [PubMed]

3. Valadi, H.; Ekström, K.; Bossios, A.; Sjöstrand, M.; Lee, J.J.; Lötvall, J.O. Exosome-mediated transfer of mRNAs and microRNAs is a novel mechanism of genetic exchange between cells. Nat. Cell Biol. 2007, 9, 654-659. [CrossRef]

4. Zhang, Y.; Liu, Y.; Liu, H.; Tang, W.H. Exosomes: Biogenesis, biologic function and clinical potential. Cell Biosci. 2019, 9, 1-18. [CrossRef]

5. Hu, Q.; Su, H.; Li, J.; Lyon, C.; Tang, W.; Wan, M.; Hu, T.Y. Clinical applications of exosome membrane proteins. Precis. Clin. Med. 2020, 3, 54-66. [CrossRef] [PubMed]

6. Hromada, C.; Mühleder, S.; Grillari, J.; Redl, H.; Holnthoner, W. Endothelial extracellular vesicles-promises and challenges. Front. Physiol. 2017, 8, 275. [CrossRef]

7. Simpson, R.J.; Lim, J.W.E.; Moritz, R.L.; Mathivanan, S. Exosomes: Proteomic insights and diagnostic potential. Expert Rev. Proteom. 2009, 6, 267-283. [CrossRef] 
8. Vlassov, A.V.; Magdaleno, S.; Setterquist, R.; Conrad, R. Exosomes: Current knowledge of their composition, biological functions, and diagnostic and therapeutic potentials. Biochim. Biophys. Acta Gen. Subj. 2012. [CrossRef]

9. Zhou, H.; Yuen, P.S.T.; Pisitkun, T.; Gonzales, P.A.; Yasuda, H.; Dear, J.W.; Gross, P.; Knepper, M.A.; Star, R.A. Collection, storage, preservation, and normalization of human urinary exosomes for biomarker discovery. Kidney Int. 2006, 69, 1471-1476. [CrossRef] [PubMed]

10. Brennan, K.; Martin, K.; FitzGerald, S.P.; O'Sullivan, J.; Wu, Y.; Blanco, A.; Richardson, C.; Mc Gee, M.M. A comparison of methods for the isolation and separation of extracellular vesicles from protein and lipid particles in human serum. Sci. Rep. 2020, 10. [CrossRef]

11. Ibsen, S.D.; Wright, J.; Lewis, J.M.; Kim, S.; Ko, S.Y.; Ong, J.; Manouchehri, S.; Vyas, A.; Akers, J.; Chen, C.C.; et al. Rapid Isolation and Detection of Exosomes and Associated Biomarkers from Plasma. ACS Nano 2017. [CrossRef] [PubMed]

12. Maroto, R.; Zhao, Y.; Jamaluddin, M.; Popov, V.L.; Wang, H.; Kalubowilage, M.; Zhang, Y.; Luisi, J.; Sun, H.; Culbertson, C.T.; et al. Effects of storage temperature on airway exosome integrity for diagnostic and functional analyses. J. Extracell. Vesicles $\mathbf{2 0 1 7}, 6$. [CrossRef] [PubMed]

13. Ludwig, N.; Whiteside, T.L.; Reichert, T.E. Challenges in exosome isolation and analysis in health and disease. Int. J. Mol. Sci. 2019, 20, 4684. [CrossRef] [PubMed]

14. Muller, L.; Hong, C.S.; Stolz, D.B.; Watkins, S.C.; Whiteside, T.L. Isolation of biologically-active exosomes from human plasma. J. Immunol. Methods 2014, 411, 55-65. [CrossRef] [PubMed]

15. Serrano-Pertierra, E.; Oliveira-Rodríguez, M.; Rivas, M.; Oliva, P.; Villafani, J.; Navarro, A.; Blanco-López, M.C.; CernudaMorollón, E. Characterization of plasma-derived extracellular vesicles isolated by different methods: A comparison study. Bioengineering 2019, 6, 8. [CrossRef]

16. Théry, C.; Amigorena, S.; Raposo, G.; Clayton, A. Isolation and Characterization of Exosomes from Cell Culture Supernatants and Biological Fluids. Curr. Protoc. Cell Biol. 2006. [CrossRef] [PubMed]

17. Welton, J.L.; Webber, J.P.; Botos, L.A.; Jones, M.; Clayton, A. Ready-made chromatography columns for extracellular vesicle isolation from plasma. J. Extracell. Vesicles 2015, 4, 1-9. [CrossRef] [PubMed]

18. Tauro, B.J.; Greening, D.W.; Mathias, R.A.; Ji, H.; Mathivanan, S.; Scott, A.M.; Simpson, R.J. Comparison of ultracentrifugation, density gradient separation, and immunoaffinity capture methods for isolating human colon cancer cell line LIM1863-derived exosomes. Methods 2012, 56, 293-304. [CrossRef]

19. Yu, L.L.; Zhu, J.; Liu, J.X.; Jiang, F.; Ni, W.K.; Qu, L.S.; Ni, R.Z.; Lu, C.H.; Xiao, M.B. A Comparison of Traditional and Novel Methods for the Separation of Exosomes from Human Samples. Biomed Res. Int. 2018, 1-9. [CrossRef]

20. Tian, Y.; Gong, M.; Hu, Y.; Liu, H.; Zhang, W.; Zhang, M.; Hu, X.; Aubert, D.; Zhu, S.; Wu, L.; et al. Quality and efficiency assessment of six extracellular vesicle isolation methods by nano-flow cytometry. J. Extracell. Vesicles 2020, 9. [CrossRef]

21. Livshts, M.A.; Khomyakova, E.; Evtushenko, E.G.; Lazarev, V.N.; Kulemin, N.A.; Semina, S.E.; Generozov, E.V.; Govorun, V.M. Isolation of exosomes by differential centrifugation: Theoretical analysis of a commonly used protocol. Sci. Rep. 2015, 5. [CrossRef]

22. Jeppesen, D.K.; Hvam, M.L.; Primdahl-Bengtson, B.; Boysen, A.T.; Whitehead, B.; Dyrskjøt, L.; Ørntoft, T.F.; Howard, K.A.; Ostenfeld, M.S. Comparative analysis of discrete exosome fractions obtained by differential centrifugation. J. Extracell. Vesicles 2014, 3. [CrossRef]

23. Szatanek, R.; Baran, J.; Siedlar, M.; Baj-Krzyworzeka, M. Isolation of extracellular vesicles: Determining the correct approach (review). Int. J. Mol. Med. 2015, 36, 11-17. [CrossRef] [PubMed]

24. Lobb, R.J.; Becker, M.; Wen, S.W.; Wong, C.S.F.; Wiegmans, A.P.; Leimgruber, A.; Möller, A. Optimized exosome isolation protocol for cell culture supernatant and human plasma. J. Extracell. Vesicles 2015, 4. [CrossRef] [PubMed]

25. Sui, X.; Arlt, H.; Brock, K.P.; Lai, Z.W.; DiMaio, F.; Marks, D.S.; Liao, M.; Farese, R.V.; Walther, T.C. Cryo-electron microscopy structure of the lipid droplet-formation protein seipin. J. Cell Biol. 2018, 217, 4080-4091. [CrossRef] [PubMed]

26. Wu, D.; Yan, J.; Shen, X.; Sun, Y.; Thulin, M.; Cai, Y.; Wik, L.; Shen, Q.; Oelrich, J.; Qian, X.; et al. Profiling surface proteins on individual exosomes using a proximity barcoding assay. Nat. Commun. 2019, 10. [CrossRef] [PubMed]

27. Larssen, P.; Wik, L.; Czarnewski, P.; Eldh, M.; Lof, L.; Goran Ronquist, K.; Dubois, L.; Freyhult, E.; Gallant, C.J.; Oelrich, J.; et al. Tracing cellular origin of human exosomes using multiplex proximity extension assays. Mol. Cell. Proteom. 2017, 16, 502-511. [CrossRef] [PubMed]

28. Trusted Exosome Purification. Available online: https://www.cellgs.com/products/exo-spinand8482-mini-hd-columns.html (accessed on 20 January 2021).

29. Mørk, M.; Handberga, A.; Pedersen, S.; Jørgensen, M.M.; Bæk, R.; Nielsen, M.K.; Kristensen, S.R. Prospects and limitations of antibody-mediated clearing of lipoproteins from blood plasma prior to nanoparticle tracking analysis of extracellular vesicles. $J$. Extracell. Vesicles 2017. [CrossRef] [PubMed]

30. Sódar, B.W.; Kittel, Á.; Pálóczi, K.; Vukman, K.V.; Osteikoetxea, X.; Szabó-Taylor, K.; Németh, A.; Sperlágh, B.; Baranyai, T.; Giricz, Z.; et al. Low-density lipoprotein mimics blood plasma-derived exosomes and microvesicles during isolation and detection. Sci. Rep. 2016. [CrossRef]

31. Yuana, Y.; Levels, J.; Grootemaat, A.; Sturk, A.; Nieuwland, R. Co-isolation of extracellular vesicles and high-density lipoproteins using density gradient ultracentrifugation. J. Extracell. Vesicles 2014, 3. [CrossRef] [PubMed] 
32. Dragovic, R.A.; Gardiner, C.; Brooks, A.S.; Tannetta, D.S.; Ferguson, D.J.P.; Hole, P.; Carr, B.; Redman, C.W.G.; Harris, A.L.; Dobson, P.J.; et al. Sizing and phenotyping of cellular vesicles using Nanoparticle Tracking Analysis. Nanomed. Nanotechnol. Biol. Med. 2011, 7, 780-788. [CrossRef]

33. German, J.B.; Smilowitz, J.T.; Zivkovic, A.M. Lipoproteins: When size really matters. Curr. Opin. Colloid Interface Sci. 2006, 11, 171-183. [CrossRef]

34. Lei, D.; Yu, Y.; Kuang, Y.L.; Liu, J.; Krauss, R.M.; Ren, G. Single-molecule 3D imaging of human plasma intermediate-density lipoproteins reveals a polyhedral structure. Biochim. Biophys. Acta Mol. Cell Biol. Lipids 2019, 1864, 260-270. [CrossRef]

35. Yu, Y.; Kuang, Y.L.; Lei, D.; Zhai, X.; Zhang, M.; Krauss, R.M.; Ren, G. Polyhedral 3D structure of human plasma very low density lipoproteins by individual particle cryo-electron tomography. J. Lipid Res. 2016, 57, 1879-1888. [CrossRef] [PubMed]

36. Zhang, L.; Song, J.; Cavigiolio, G.; Ishida, B.Y.; Zhang, S.; Kane, J.P.; Weisgraber, K.H.; Oda, M.N.; Rye, K.A.; Pownall, H.J.; et al. Morphology and structure of lipoproteins revealed by an optimized negative-staining protocol of electron microscopy. J. Lipid Res. 2011, 52, 175-184. [CrossRef] [PubMed]

37. Frühbeis, C.; Helmig, S.; Tug, S.; Simon, P.; Krämer-Albers, E.M. Physical exercise induces rapid release of small extracellular vesicles into the circulation. J. Extracell. Vesicles 2015, 4. [CrossRef]

38. Gardiner, C.; Ferreira, Y.J.; Dragovic, R.A.; Redman, C.W.G.; Sargent, I.L. Extracellular vesicle sizing and enumeration by nanoparticle tracking analysis. J. Extracell. Vesicles 2013, 2. [CrossRef]

39. Bachurski, D.; Schuldner, M.; Nguyen, P.H.; Malz, A.; Reiners, K.S.; Grenzi, P.C.; Babatz, F.; Schauss, A.C.; Hansen, H.P.; Hallek, M.; et al. Extracellular vesicle measurements with nanoparticle tracking analysis-An accuracy and repeatability comparison between NanoSight NS300 and ZetaView. J. Extracell. Vesicles 2019, 8. [CrossRef] 